\title{
La hipótesis de parentesco Guaicurú-Mataguayo: estado actual de la cuestión
}

\author{
J. Pedro Viegas Barros ${ }^{1}$
}

\section{Resumen}

Entre las lenguas de dos de las más importantes familias lingüísticas del Gran Chaco, guaicurú y mataguayo, existen sin duda transferencias léxicas y gramaticales, calcos semánticos y otras influencias, resultado del contacto lingüístico y de la difusión areal. Pero, por otra parte, hay muy pocas semejanzas en el vocabulario llamado cultural (nombres de plantas y animales de interés especial, herramientas, etc.) y hay - en cambio - una cantidad considerable de similitudes en el léxico básico (por ejemplo: verbos de movimiento, nombres de partes del cuerpo, etc), así como un gran isomorfismo gramatical; y estas semejanzas parecen ajustarse a correspondencias fonológicas regulares. Como resultado de todo ello, la hipótesis de parentesco guaicurú-mataguayo parece, en el estado actual de la investigación, sumamente prometedora.

Palabras-clave: Guaicurú. Mataguayo. Lingüística histórica. Hipótesis de Parentescos Distantes.

\begin{abstract}
Two of the most important language families of the Gran Chaco, Guaykuruan and Mataguayan, display undeniable lexical and grammatical transferences, semantic calques and other influences, as a result of linguistic contact and areal diffusion. But, on the other hand, there are very few resemblances in the so-called cultural vocabulary (names of plants and animals of special interest, tools, etc.), and, by the contrary, there is a considerable amount of similarities in the nuclear lexicon (for example: verbs of movement, names of body parts, etc), as well as an important grammatical isomorphism; and these resemblances seem to be tied by systematic phonological correspondences. In reason of all this, the relationship of Guaykuruan and Mataguayan appears to be, in the current state of the research, a very promising hypothesis.
\end{abstract}

Keywords: Guaykuruan. Mataguayan. Historical Linguistics. Remote Relationship Hypotheses.

1 CONICET / Instituto de Lingüística, UBA, 25 de Mayo 217, 1er. Piso (1002) Ciudad Autónoma de Buenos Aires, Argentina. E-mail: peviegas@gmail.com. 


\section{Las familias guaicurú y mataguaya}

\subsection{La familia guaicurú}

La familia lingüística guaicurú incluye las lenguas kadiweu hablado en el estado brasileño de Mato Grosso do Sul, el pilagá en la provincia argentina de Formosa, el toba en la región del Chaco paraguayo (principalmente en el departamento de Presidente Hayes) y en las provincias argentinas de Salta, Formosa, Chaco y Santa Fe, y el mocoví, en estas dos últimas provincias. También pertenecían a esta familia las lenguas extintas mbayá (del Chaco paraguayo) y abipón, que se habló principalmente en la provincia de Santa Fe. Kadiweu y mbayá forman la rama septentrional de la familia, las demás lenguas la rama meridional. Dentro de estas, mocoví, pilagá y toba forman el grupo qom. Y dentro del grupo qom, a su vez, el pilagá y el toba son las lenguas más cercanamente emparentadas entre sí. La subclasificación de esta familia, entonces, es la siguiente:

1. Guaicurú del Norte ${ }^{2}$

\subsection{Guaicurú del Norte

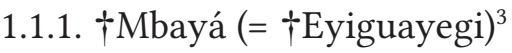 \\ 1.1.2. Kadiweu}

2. Guaicurú del Sur

2.1. †Abipón

2.2. Qom

\subsubsection{Mocoví}

2.2.2. Pilagá-toba

\subsubsection{Pilagá}

2.2.2.2. $\mathrm{Toba}^{4}$

\footnotetext{
2 En realidad, mbayá y kadiweu son dialectos de una misma lengua: en el presente trabajo se citarán para el guaicurú del Norte tanto formas no fonologizadas del mbayá (debido a que el léxico publicado de esta variedad es mucho más extenso) como formas en notación fonológica del kadiweu.

$3 \mathrm{El}$ signo $\uparrow$ delante de un nombre de lengua indica que la misma está extinguida.

4 Otras dos lenguas extintas y poco conocidas, el guachí y el payaguá, a menudo clasificadas como miembros de la familia guaicurú, no serán consideradas en el presente trabajo.

El payaguá fue una lengua hablada por un pueblo canoero a lo largo del río Paraguay (principalmente, del Alto Paraguay). La última hablante falleció en el año 1940. Se conoce una decena de pequeños vocabularios, de los siglos XVIII a XX, con un corpus total de unos 600 lexemas distintos. Dentro de lo poco que se sabe de su gramática, se encuentran algunas notables semejanzas con lenguas guaicurúes y mataguayas, como:

(Boggiani) $<\boldsymbol{i}->$ ' $1^{\text {a }}$ p. sing. posesiva', cf. la serie (96)

(Boggiani) $<\boldsymbol{l}->$. ' $3^{\mathrm{a}}$ p. sing. posesiva', cf. la serie (102).

(Boggiani) <-am> tema común a los distintos pronombres personales, cf. las series (80) a
} 


\subsection{La familia mataguaya}

La familia lingüística mataguayo, por su parte, incluye cuatro lenguas: maká, originaria del Chaco paraguayo (departamento Presidente Hayes) y hoy hablada principalmente en una colonia en la ciudad de Asunción; niwaklé (también llamada chulupí o ajlujlay), hablada en los departamentos de Boquerón y Presidente Hayes en Paraguay y en la provincia de Salta en Argentina; chorote en la provincia argentina de Salta y en el departamento paraguayo

(82).

Sin embargo, las semejanzas léxicas detectadas hasta el momento son pocas, quizás no más de un $10 \%$ de todo el material payaguá documentado. Algunas de estas semejanzas son con certeza préstamos en una u otra dirección, por ejemplo:

(Schmidt) < pagEsÉne>, cf. kadiweu bigitfe:na 'gato',

(Schmidt) < n'Ets-Ék>, cf. maká naats'axik 'maíz',

si bien muchas de las similitudes léxicas se encuentran más bien en el ámbito no cultural: (Cerviño [Mitre 1910]) < $\boldsymbol{m a}>$ ' 'id', cf. la serie (19) del presente trabajo,

(Schmidt) $<-A \boldsymbol{k} \hat{A} \boldsymbol{l}>$ 'lengua', cf. la serie (56) del presente trabajo,

(Boggiani 1900) <-ácc, - $\boldsymbol{a}_{\dot{e}} \boldsymbol{c c}>$ 'labio inferior', cf. proto-pilagá-toba *áka (> pilagá -ak, toba antiguo $<-\boldsymbol{a c} \boldsymbol{c}>$, Toba $-\boldsymbol{a k}$ ) 'labio inferior',

(Boggiani) <-ái > 'querer', cf. proto-mataguayo I *-haju (> maká -(h)eju, -(h)iju, niwaklé -xaju 'sufijo desiderativo', etc.

La lengua guachí, por su parte, fue hablada por un pequeño grupo que a mediados del siglo XIx vivía en las cabeceras del río Paraguay, en cercanías de la actual ciudad de Miranda (Mato Grosso do Sul, Brasil), y se extinguó en la segunda mitad de ese siglo. Es conocida por un vocabulario recogido en 1845 (Castelnau 1851:278-280), de unos 145 ítems. Unos pocos datos más - hasta ahora inéditos - se encuentran en los manuscritos de Natterer (Adelaar 2012 y comunicación personal). Una o dos características gramaticales y algunas decenas de ítems del vocabulario de Castelnau, muchos de ellos no pertenecientes al léxico cultural, presentan semejanzas con lenguas guaicurúes:

$<\boldsymbol{a g}->$ : kadiweu $\boldsymbol{a} G$ - 'prefijo negativo',

$<$ euaité> 'demasiado': kadiweu owidi 'mucho',

$<$ aney> : abipón noai 'pez', etc.,

con lenguas mataguayas:

<leek> 'tierra' : maká 17ekhip 'polvo',

$<$ ia-note $>$ : chorote -xnatowo -xnatawe 'nariz', etc.,

o con lenguas de ambas familias al mismo tiempo:

$<\boldsymbol{i i k}>$ 'comer', cf. la serie (13) del presente trabajo,

$<\boldsymbol{a m m a}>$ 'dormir', cf. la serie (22) del presente trabajo,

$<\boldsymbol{e u}$-echo> 'dos', cf. la serie (39) del presente trabajo, etc,.

En conjunto, las semejanzas del payaguá y el guachí con las lenguas de las familias guaicurú y mataguaya (aunque algunas pueden ser sugerentes) son pocas y no parecen suficientes para aseverar con certeza que haya --en este caso-- parentesco. Lo que sí parece claro es que, si efectivamente existiera tal relación, el guachí y el payaguá no pertenecerían ni a la familia guaicurú ni a la mataguaya (cf. Viegas Barros 1992-1993, 2004). 
de Boquerón, y wichi (o mataco), hablada en las provincias argentinas de Chaco, Formosa y Salta, así como en el departamento boliviano de Tarija. La subclasificación de esta familia todavía no está establecida adecuadamente. De acuerdo a Fabre (2005; cf. también Campbell y Grondona 2007:23, nota 14), la subclasificación más probable sería la siguiente:

1. Mataguayo I

1.1. Maká

1.2. Niwaklé

2. Mataguayo II

2.1. Chorote

2.2. Wichí

En el presente trabajo se adoptará esta clasificación interna de la familia. Según la misma, basta que una serie tenga al menos un cognado en una lengua mataguaya I y otro en una lengua mataguaya II para reconstruir una forma ancestral en proto-mataguayo. Hay que señalar, sin embargo, que Fabre advierte que habría que revisar la posición del chorote, dado que si bien esta lengua parece muy próxima del wichí en lo que respecta al léxico, estaría - sin embargo - más cercana al niwaklé y al maká en algunos importantes aspectos gramaticales.

\section{Los sistemas fonológicos proto-guaicurú y proto- mataguayo}

\subsection{Proto-guaicurú}

Mi reconstrucciòn actual del sistema fonológico proto-guaicurú (Viegas Barros 2013, en prensa) es la siguiente:

Consonantes:

$\begin{array}{ll}{ }^{*} p, & { }^{*} t \\ { }^{*} p & { }^{*} t, \\ & { }^{*} d \\ & { }^{*} d, \\ { }^{*} w & { }^{*} l \\ { }^{*} w & { }^{*} l \\ { }^{*} m & { }^{*} n \\ { }^{*} m, & { }^{*} n,\end{array}$
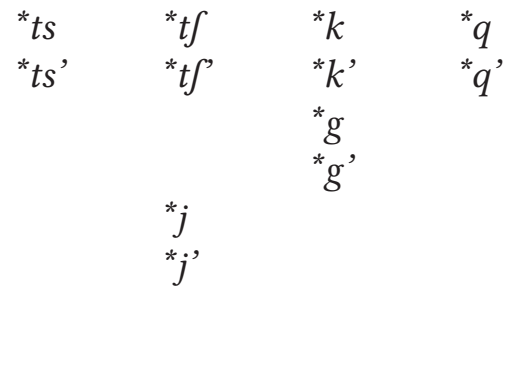


\section{Vocales:}

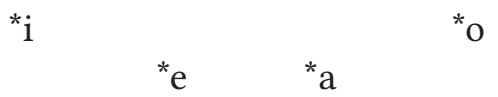

Las consonantes laringalizadas, indicadas en este trabajo mediante el diacrítico' tras la letra correspondiente, habrían sido fonéticamente postglotalizadas (eyectivas) en el caso de las obstruyentes sordas, preglotalizadas (inyectivas) en el caso de las obstruyentes sonoras, y preaspiradas en el caso de las aproximantes y nasales ${ }^{5}$.

Los principales cambios que habrían ocurrido en $\mathrm{PGS}^{6}$ son (1) la desaparición de las africadas (por defricativización en el caso de las alveolares: PG ${ }^{*} t \boldsymbol{s}$, " $t \boldsymbol{s}$ '> PGS ${ }^{*} \boldsymbol{t}$-; y -por el contrario- deoclusivización en el caso de las palatales: PG ${ }^{*} t \boldsymbol{t},{ }^{*} \boldsymbol{t} \boldsymbol{t}$ ' > PGS ${ }^{*} \boldsymbol{s}$ ), (2) la fusión de casi todas las consonantes simples y laringalizadas en el caso de las obstruyentes sordas y aproximantes:

5 La evolución de mi reconstrucción de la fonología del proto-guaicurú puede seguirse a lo largo varios trabajos (Viegas Barros 1992-1993, 2004, 2006, 2008, 2010, 2013, en prensa). Una reconstrucción bastante diferente se encuentra en Ceria y Sandalo (1995).

6 Las abreviaturas usadas para nombres de lenguas y prootolenguas son: Ab Abipón, Cho chorote, Ka Kadiweu, Mb mbayá, Mk maká, Mo mocoví, Ni niwaklé, Pi pilagá, PG protoguaicurú, PGS proto-guaicurú del sur, PM proto-mataguayo, PM1 proto-mataguayo I, PM2 proto-mataguayo II, PPT proto-pilagá-toba, PQ proto-qom, To toba, ToAnt toba antiguo, Wi Wichí.

Las abreviaturas de las fuentes utilizadas para cada lengua son las siguientes:

Ab: Najlis (1966).

Cho: Gerzenstein (1979, 1983), Drayson (2010), Carol (2011; Salanova y Carol 2011).

Ka: Griffiths (2002), Sandalo (1997).

Mb: Sánchez Labrador (1971, 1972).

Mk: Belaieff (1940), Gerzenstein (1999).

Mo: Tavolini (Lafone Quevedo 1892-1893), Paucke (1942-1944), Grondona (1998), Gualdieri (1998), Buckwalter y Litwiller de Buckwalter (2004).

Ni: Seelwische (1975), Stell (1989), Stell y Seelwische (2000).

Pi: Arenas (1993), Buckwalter y Litwiller de Buckwalter (2004), Vidal (2001, 2006, 2011).

To: Buckwater (1980), Buckwalter y Litwiller de Buckwalter (2004) Censabella (2002), Messineo (2003).

ToAnt: Barcena (Lafone Quevedo 1894-1896, 1896b).

Wi: Alvarsson (1984), Braunstein (2000), Claesson (1994), Tovar (1981), Viñas Urquiza (1974).

Cuando no se menciona variedad del Cho, se trata del dialecto yófwaja. Para el Wi se ha tomado como el dialecto por default a la variedad del Pilcomayo salteño descripta por Viñas Urquiza.

Remplazo algunos símbolos utilizados por los distintos autores, a fin de homogeneizar las notaciones fonológicas.

Los signos <> encierran grafías prefonológicas originales. Otros signos empleados son: V vocal no especificada, $\mathrm{C}$ consonante no especificada. 
PG ' $\boldsymbol{p}$ ' y ${ }^{*} \boldsymbol{p}>$ PGS ${ }^{*} \boldsymbol{p}, \mathrm{PG}{ }^{*} \boldsymbol{w} \boldsymbol{y}^{\boldsymbol{*}} \boldsymbol{w}>\mathrm{PGS}{ }^{*} \boldsymbol{w}$, etc., pero no en el caso de las obstruyentes sonoras (caso en que se dieron las evoluciones PG ${ }^{*} \boldsymbol{d}>\mathrm{PGS}{ }^{*} \boldsymbol{d}$ pero PG ${ }^{*} \boldsymbol{d}$ ' > PGS ${ }^{*} \boldsymbol{d}$ '; PG ${ }^{*} \boldsymbol{g}>$ PGS ${ }^{*}$ ? pero PG ${ }^{*} \boldsymbol{g}$ '> PGS ${ }^{*} \boldsymbol{g}$ ), (3) en posición intervocálica, sonorización de las oclusivas dorsales sordas y deglotalización de oclusivas dorsales sordas laringalizadas (PG ${ }^{*} \boldsymbol{k}>\mathrm{PGS}{ }^{*} \boldsymbol{g}$, PG ${ }^{*} \boldsymbol{k}$ ' $>$ PGS ${ }^{*} \boldsymbol{k}$; PG ${ }^{*} \boldsymbol{q}>$ PGS ${ }^{*} G$, PG ${ }^{*} \boldsymbol{q}^{\prime}>$ PGS ${ }^{*} \boldsymbol{q}$ ), (4) la aparición de una consonante labial labiovelarizada ${ }^{*} \boldsymbol{p}^{w}$. Como resultado de estos cambios, el sistema fonológico reconstruible para el PGS es

Consonantes:

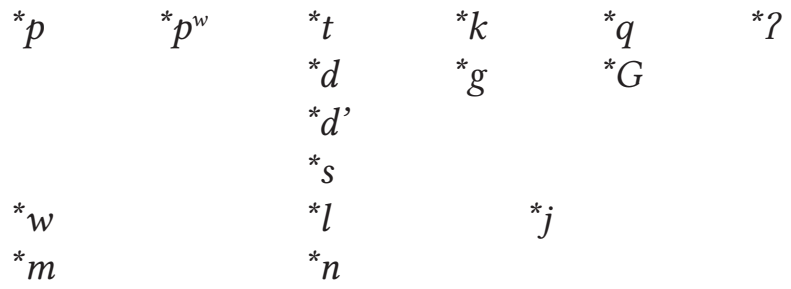

\section{Vocales:}

${ }^{*} i \quad{ }^{*} e \quad{ }^{*} a$

El sistema postulable para el nivel PQ es el mismo que para el PGS. El PPT tenía un fonema menos, debido a la fusión $\mathrm{PQ}{ }^{*} \boldsymbol{d},{ }^{*} \boldsymbol{d}>\mathrm{PPT}{ }^{*} \boldsymbol{d}$.

\subsection{Proto-mataguayo}

Mi reconstrucción actual del sistema fonológico del proto-mataguayo es la siguiente ${ }^{7}$ :

\section{Consonantes:}

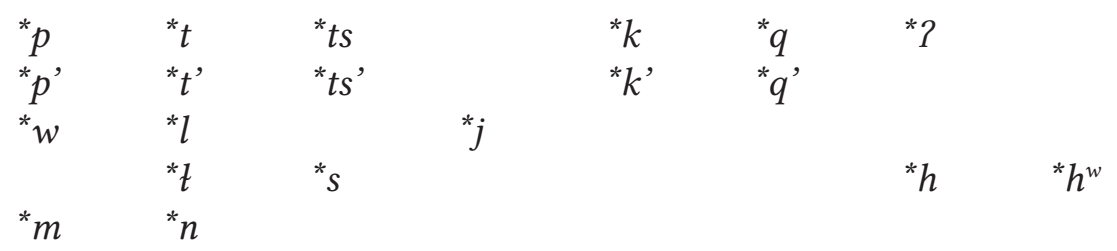

7 Reconstrucción reformulada a partir de trabajos previos (Viegas Barros 1992-1993, 2002, 2004). Una reconstrucción en gran parte distinta puede verse en Najlis (1984).

Algunas ideas sobre la prehistoria ds las vocales del wichí fueron desarrolladas por Najlis (1971). Gerzenstein (1987) y Campbell y Grondona (2007) proveen ejemplos de reconstrucciçon interna en chorote y en niwaklé, respectivamente. Gerzenstein (2003) y Censabella (2009) estudian correspondencias fonológicas y fonéticas entre variedades de la lengua wichí. 


\section{Vocales:}

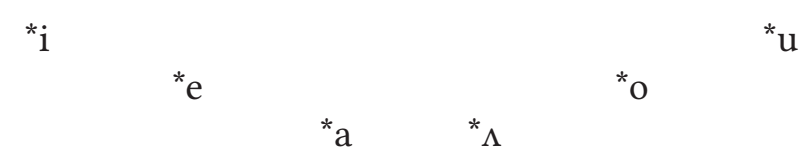

El aspecto más polémico de esta reconstrucción es, posiblemente, la postulación de los puntos de articulación velar y posvelar para las oclusivas dorsales. Campbell y Grondona (2007:18) sugieren que la oposición original pudo haber sido más bien entre " ${ }^{\star} \boldsymbol{k}^{y}$ or some kind of fronted $\boldsymbol{k}^{\text {" y }}{ }^{*} \boldsymbol{k}$.

Al parecer, sistemas idénticos o muy parecidos al proto-mataguayo se pueden reconstruir tanto para el proto-mataguayo I como para el protomataguayo II.

\section{Difusión entre mataguayo y guaicurú}

\subsection{Breve historia de los puntos de vista difusionistas}

Algunos autores han argumentado que la difusión es la causa - o la principal causa - de las semejanzas que se notan entre las lenguas de ambas familias. Así, por ejemplo, para Lafone Quevedo (1896a:137-138) y para Henry (1939) el hecho de que las similitudes que encontraron entre mataguayo y guaicurú eran sobre todo gramaticales mientras que - según ellos - el vocabulario sería en su mayor parte muy distinto, implicaría que los parecidos se debían a préstamo.

Hunt (1915:238) aplicó la noción de lenguas mixtas a las lenguas chaqueñas para intentar explicar las semejanzas guaicurú-mataguayas. Otros autores acudieron a la misma idea (por ejemplo, Tovar 1981:20, Braunstein y Martín 1990-1991, Braunstein 1992-1993, 1996, entre otros). Sin embargo, las similitudes que existen entre lenguas de ambas familias no parecen ser del tipo de las que normalmente ocurren en situaciones de mezcla de lenguas (Campbell y Grondona 2012:637-638).

En varios trabajos, Tovar (1951, 1961:35-36; 1964:376-377; 1981:20, 22; Tovar y Larrucea de Tovar 1984:54) desarrolló la idea de que la gran distancia tipológica que existiría entre guaicurú y mataguayo demuestra que ambas familias no pueden estar emparentadas, y - por tanto - cualquier semejanza detectada entre ellas debía ser explicada como resultado de difusión.

Los estudios actuales de difusión en lenguas chaqueñas intentan -en su mayoría- determinar si la región del Gran Chaco conforma un área lingüística, y procuran determinar cuáles serían los elementos tipológicos que caracterizarían a esta área, así como cuáles lenguas formarían parte de la misma y cuáles no. Entre otros aportes en esta línea de investigaciones, 
se puede citar a Kirtchuk (1996), Gerzenstein y Gualdieri (2003), Campbell (2006), Grondona (2006), Fabre (2006, 2007), Vidal (2006, 2011), Messineo y Cúneo (2007, 2010), Messineo y Gerzenstein (2007), Nonato y Sandalo (2007), Messineo (2008, 2011), Cúneo et al. (2009), Censabella y Terraza (2010), Comrie et al. (2010), Vidal y Gutiérrez (2010), Vidal (2011), Campbell y Grondona (2012), Messineo et al. (2012).

\subsection{Préstamos en el léxico cultural}

La inmensa mayoría de las semejanzas entre términos guaicurúes y mataguayos dentro del llamado "vocabulario cultural" se deben - sin duda - a préstamo. Sin embargo, parecen ser relativamente muy pocas las similitudes en esta parte del vocabulario que incluyen formas reconstruibles independientemente tanto para el proto-guaicurú como para el protomataguayo (o para algún nivel intermedio dentro de ambas familias), como ocurriría en los ejemplos (i) a (v). En algunos de estos casos - (iii) a (v) - las semejanzas se extienden a lenguas de otras familias:

(i) G: $\mathrm{PG}^{*}$ nawjék'tubérculo similar a mandioca'>Ka ene: wigigi'mandioca', Ab neiek-a (plural) 'raíces comestibles', Pi $\boldsymbol{n a}(\boldsymbol{w}) \mathbf{j o ́} \boldsymbol{k}$ 'sacha lazo' (Odontocarya asarifolia)-

M: PM *newak > Ni nowok 'mandioca silvestre', Cho niwakiwa? 'mandioca', Wi newok 'mandioca silvestre'.

(ii) G: PG * -Vtá-qa > Ka (pl.) odaqa 'bebidas' > Mo -ataGa 'aloja', Pi -atáGa, To - $\boldsymbol{a t a G \boldsymbol { a }}$ 'vino, bebida alcohólica en general'.

$\mathrm{M}: \mathrm{PM}^{*} \boldsymbol{-} \boldsymbol{t} \boldsymbol{t}>\mathrm{Ni}-\boldsymbol{\Lambda}$ ?t- 'bebida, chicha, alcohol', Wi $\boldsymbol{t}-\boldsymbol{\Lambda} \boldsymbol{t}$ 'bebida'.

(iii) G: $\mathrm{PG}$ * $\boldsymbol{w a j}$ ' $\boldsymbol{e}-\boldsymbol{k}>\mathrm{Mb}<\boldsymbol{g} \boldsymbol{u a y e g i}>$ 'algarroba jaspeada', Ab oai- $\boldsymbol{k}$ 'algarrobo blanco'

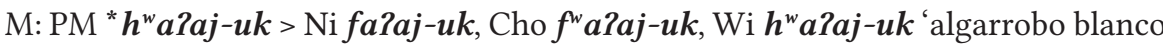
(Prosopis alba)'.

Lule-Vilela: Lule waja 'algarrobo verde negro (y su fruta)'.

(iv) G: PG * pijóko > Ka -biogo 'pan de mandioca', Pi pijók 'mandioca'.

M: PM1 *pahajak > Mk pexejek, Ni pexajak 'batata' (y cf. Wi pi?jok 'mandioca', que no parece cognado de las formas $\mathrm{Mk}$ y Ni).

Zamuco: Ayoreo peheei 'mandioca'.

Enhlet-Enenhlet: Angaité pija pihija, Enlhet pehaja?, Lengua Sur piheji ‘batata'.

(v) G: PQ *elé > Mo ele, Pi elé, To ele 'loro hablador'.

M: $\mathrm{PM}$ *ele > Ni ekle, Cho ele?, Wi Rele 'loro hablador'.

Lule-Vilela: Lule ele 'papagayo'.

En otros casos, las semejanzas están poco extendidas (a menudo sólo en una sola lengua) en una de las dos familias, cf. los ejemplos (vi) a (ix): 
(vi) G: PG *op'owé > Ka opoe, Ab hapeo (posible errata por *hapoe), Mo powe $~$ pohe, $\mathrm{Pi}$ pohé poí. 'cuervo negro (Coragyps atratus)'.

M: Mk opowi 'cuervo negro (Coragyps atratus)'.

(vii) G: $\mathrm{PQ}$ *wáloG > Mo waloq, Pi wáloq 2wáloq, To waloq 'algodón'.

M: Cho walek 'algodón'.

(viii) G: $\mathrm{PQ}{ }^{*}$-qaná > Mo -a?na, Pi -qaná, To -qana 'aguja'.

M: Wi kıno (dialecto weenhayek qano?) 'aguja'.

(ix) G: Ab-enenk 'concha, cuchara'.

M: PM *-anek > Mk n-enek 'cuchara', Wi l-anek 'concha, valva, almeja'.

Semejanzas como las precedentes son sin duda interpretables como préstamos, y en varios casos es incluso evidente cuál ha sido la dirección de los mismos: por ejemplo, del guaicurú al mataguayo en las series (vi) a (viii), del mataguayo al guaicurú en la serie (ix).

En realidad, la mayoría de los términos del vocabulario cultural son muy distintos entre lenguas de ambas familias. Como ejemplo pueden cotejarse los treinta términos siguientes en toba (guaicurú) y en wichí (mataguayo), lenguas que -por lo menos en algunas zonas- han estado en contacto durante siglos:

To -tfikn-ek, Wi -lutseh 'arco'.

To -tak-jaGaj, Wi -hun 'lanza'.

To -pon, Wi -etek-tah 'garrote'.

To -ajlon-ek, Wi tsonat 'cuchillo'.

To -qatfipe, Wi ho?san 'hacha'.

To-alegek, Wi -to-wut 'red de pesca'.

To -ogo, Wi - wuj 'ropa'.

To -sa:t-aGaki, Wi - $\boldsymbol{k}_{\boldsymbol{\Lambda}}-\boldsymbol{n} \boldsymbol{i j} \boldsymbol{a} \boldsymbol{k}$ 'cinturón, faja'.

To -apela?, Wi nisıh 'calzado'.

To -qola, Wi -tamis 'collar'.

To - $\boldsymbol{w a G a} \boldsymbol{a} \boldsymbol{f} \boldsymbol{k}$, Wi $-\boldsymbol{k}^{w} \boldsymbol{e}-\boldsymbol{h}^{w} \boldsymbol{a t}$ 'brazalete'.

To -poto-zik, Wi wesah 'tela'.

To -qotaki, Wi hilu 'bolsa'.

To qa?ajk, Wi najih 'camino'.

To -ojik, Wi hup 'casa'.

To -tet, Wi -tse $\boldsymbol{k}^{w} \boldsymbol{e k}$ 'coser'.

To -qo?ile, Wi -p'ak 'teñir'.

To -paGah-pi, Wi -potsin 'tejer'. 
To - $\mathbf{a d}$-aGan-aGaki, Wi - $\boldsymbol{h}^{w}$ elek 'mortero'.

To -t-aGaki, Wi -to-weh 'olla'.

To -?awaGa, Wi -ihpat 'maíz'.

To -kat, Wi t finah 'metal'.

To -qa?ap-aGa, Wi ihpat'-i 'chicha'.

To -imaGa-ni, Wi p'ek 'pozo'.

To -atok, Wi tsalana 'canoa'.

To - $\lambda$ igot, Wi -seklo? 'remar'.

To -qataki, Wi pum 'tambor'.

To -ata?, Wi katfa 'remedio'.

To piloGo-naq, Wi hajawu 'chamán'.

To -afi, Wi nijat 'cacique'.

Estas diferencias son congruentes con lo que se sabe acerca de la fuerte resistencia a los préstamos característica de las lenguas chaqueñas en general (cf. Campbell y Grondona 2012:653-654).

\section{La hipótesis de parentesco guaicurú-mataguayo}

\subsection{Breve historia de la hipótesis}

A fines del siglo XIX, Lafone Quevedo señaló las notables similitudes en los marcadores de persona del wichí y el toba, que lo llevaron a preguntarse si la primera de estas lenguas no sería - en sus palabras - "un dialecto degenerado del Guaycurú" (Lafone Quevedo 1896a:140), aunque finalmente se decidió, como se vió en en el apartado 3.1, a considerar estos parecidos como producto del préstamo.

A mediados del siglo pasado, Mason (1950:201) propuso el parentesco de ambas familias en un tronco lingüístico al que llamó "macro-guaicurú" (variante: "macro-guaicuruan"), dejando abierta la posibilidad de incluir en el mismo otras familias como la lule-vilela o la chiquitana.

Posteriormente Greenberg (1987:73-74) incluyó a las lenguas mataguayas y guaicurúes en un grupo denominado "macro-pano" que incluiría además a las lenguas o grupos de lenguas: lule, vilela, mosetén, maskoy, charrúa, tacana y pano. Según Greenberg la relación entre mataguayo y guaicurú sería la más estrecha que existe dentro del "macro-pano".

Kaufman (1990, 1994) comparó las clasificaciones de Swadesh (1959), Loukotka (1968), Suárez (1988 [1974]) y Greenberg (1987), y a partir de ellas estableció "racimos" (clusters) de lenguas, esto es, grupos compuestos por familias cuyo parentesco coincidieron en proponer dos o más de dichos autores: la idea es que tales posibles agrupaciones merecerían ser ulteriormente 
investigadas con algún detenimiento para ver si los parentescos propuestos coincidentemente por dos o más autores se confirman o no. A partir de esta comparación de clasificaciones, Kaufman estableció (1990:56-57) un racimo "macro-Waikuruan" compuesto por las familias mataguaya y guaicurú, además de charrúa, maskoy y lule-vilela. En el caso del guaicurú y el mataguayo, los dos antecedentes para postular su parentesco son la clasificación ya mencionada de Greenberg y la de Swadesh, quien - partiendo de cálculos léxico-estadísticos - había colocado tanto al mataguayo como al guaicurú, junto con otras lenguas, dentro de una agrupación denominada "macroMapuche". La hipótesis del racimo "macro-Waikuruan" no aparece señalada en Kaufman (1990), como sucede en otros casos, con las acotaciones al margen de "buena", "probable" o "posible", por lo que - al parecer - esta hipótesis de parentesco sería más dudosa que otras como las de las agrupaciones macroye, lule-vilela, etc. Posteriormente, Kaufman (1994:67) quitó al lule-vilela del macro-Waikuruan.

En miprimer trabajo referido al tema ${ }^{8}$, señalé que existirían correspondencias fonológicas al parecer bastante regulares entre los miembros del posible tronco lingüístico, al que denominé "mataguayo-guaycurú" (Viegas Barros 1992-1993). En aquel trabajo no llegué a una respuesta definitiva a la pregunta que daba título a mi artículo, puesto que la cantidad de formas comparadas era - a mi entender - insuficiente para ello. En Viegas Barros (2006), resalté que el isomorfismo gramatical mataguayo-guaicurú es de un tipo muy distinto al que se da en situaciones típicas de Sprachbund, y - por tanto - necesita una explicación diferente.

Grondona (2006) comparó ambas familias lingüísticas y señaló que habría una alta cantidad de semejanzas gramaticales frente a relativamente pocas semejanzas léxicas, sin llegar (como yo en mi trabajo de 1992-1993) a una respuesta definitiva a la cuestión de si se trata de semejanzas areales o hereditarias.

\subsection{Evidencias a favor de la hipótesis de parentesco guaicurú- mataguayo}

\subsubsection{Semejanzas en el léxico no cultural}

Además de semejanzas generalmente poco extendidas en algunos términos culturales, entre las lenguas guaicurúes y mataguayas también existen similitudes - por lo común ampliamente distribuidas - en formas pertenecientes al léxico no cultural. Si todas las semejanzas entre guaicurú y mataguayo fuesen como las presentadas en los ejemplos (i) a (ix) de la sección 3.2, sería necesario concluir que las similitudes entre ambas

8 Un trabajo muy preliminar. Considero ahora que aproximadamente un $10 \%$ de las comparaciones efectuadas en él resultan -por una u otra razón- inválidas. 
familias se deberían exclusivamente a difusión. Pero lo cierto es que existe una cantidad verdaderamente notable de semejanzas en el léxico básico, así como en ítems gramaticales; y estas similitudes se encuentran tan extendidas como para que muchos de los ítems involucrados puedan ser reconstruidos independientemente tanto en proto-guaicurú como en proto-mataguayo (o en algún nivel intermedio dentro de una o de ambas familias).

Entre estas semejanzas léxicas guaicurú-mataguayo ${ }^{9}$ se encuentran términos pertenecientes a los siguientes campos semánticos:

\section{Acciones, procesos, eventos}

(1) G: PG *á́jo > Ka -ajo:, Mo -jo, Pi -ájo, To -ajo 'volar'.

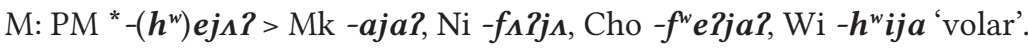

(2) G: PG ${ }^{*}-\boldsymbol{a l V}\left(\mathrm{o}^{*}-\boldsymbol{a l}\right.$ ' $)$ > Ka -ale-gi 'quemar', -alo-Go 'incendiar el campo', $\mathrm{Ab}$-alah-ini 'arder”.

M: PM1 *-at> Mk -at-ets, -at-ixul'calentarse', -at-ixup 'calentar', -et-xu?'quemar (algo / a alguien)', Ni -at-etf'quemazón'.

(3) G: PG ${ }^{*}-\boldsymbol{a m}{ }^{\prime}-\boldsymbol{a} q \boldsymbol{a} \boldsymbol{~ > ~ K a ~ - a m : a G a , ~ M o ~ - ( a ) m a q , ~ P i ~ - a m a ́ q , ~ T o ~ - a m a q , ~ - a m a G a - ~}$ 'empujar'

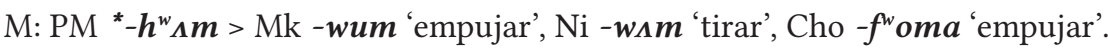

(4) G: PG * $-\boldsymbol{a n}>\mathrm{Ka}-\boldsymbol{a n}-\boldsymbol{t} \boldsymbol{i}$ 'plantar', Ab - $\boldsymbol{a} \boldsymbol{n}-\boldsymbol{i}$, Mo - $\boldsymbol{a n}$, Pi - $\boldsymbol{a n}$, To - $\boldsymbol{a n}$ '

$\mathrm{M}: \mathrm{PM}{ }^{*}$-an $>\mathrm{Mk}-\boldsymbol{e n}-\boldsymbol{i}, \mathrm{Ni}-\boldsymbol{a n}$, Cho -en -exn 'poner'.

(5) $\mathrm{G}: \mathrm{PG}{ }^{*}-\boldsymbol{a p} \boldsymbol{p}^{\prime} \boldsymbol{a}>\mathrm{Mb}<-\boldsymbol{a p a} \boldsymbol{a}, \mathrm{Ab}-\boldsymbol{a p}$ 'sufrir'.

$\mathrm{M}: \mathrm{PM}{ }^{*}-\boldsymbol{a p}>\mathrm{Mk}-\boldsymbol{a p}$, Ni - ap, Cho - ap 'llorar', Wi -ip 'gritar'.

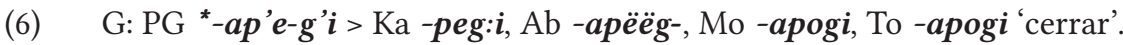

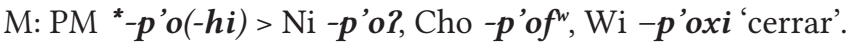

(7) G: PG ${ }^{*}-\boldsymbol{a p}$ 'i > Ka -api-pe-n 'chupar', Mb <-api tibibi $>$ 'relamerse', Ab -apii, To -api-tfigi, -api-lek, -apj-oGon 'lamer'

M: PM *-n-ap'u > Ni -na?pu?-k, Cho -napu-tsi, (dialectos yowuwa y manjuy) -napu?, Wi -nap'u 'chupar'.

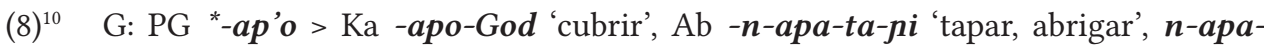
Rek 'cobertura', 'encerrado, tapado', -n-apa-ta-gi 'cerrado'; Mo -apo-lek, -apo:ta-lek, -apo-Pgit 'tapar', Pi -apó-t $f$ i:-ni 'tapar', -apó? 'techo', To -apo-Gon, -apolek, -apo-Poget 'tapar', -apo-Got 'tapa', -apo? 'techo'.

M: PM * ${ }^{*}(\boldsymbol{a}) \boldsymbol{p}^{\prime} \boldsymbol{o}-\boldsymbol{t}>\mathrm{Ni}-\boldsymbol{a p}$ 'o?t, Cho $-\boldsymbol{p}$ 'ot, Wi -p'ot 'tapa'.

9 Los significados de los eventuales cognados guaicurú-mataguayos presentados en este trabajo no son siempre los mismos en las lenguas de ambas familias, pero se encuentran dentro del rango de posibilidades de deslizamiento semántico esperable entre lenguas cuyo parentesco podría remontar a varios milenios.

10 En ambas familias, la raíz es la misma que la de la serie (6). 
(9) G: $\mathrm{PG}{ }^{*}-a q$ 'atf'in > $\mathrm{Mb}<-$ acachini $>$, Mo -aqafin, $\mathrm{Pi}$-aqasén, To -qafin 'estornudar'.

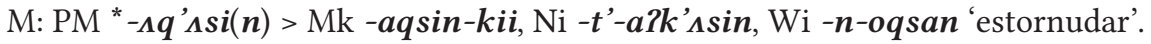

(10) G: PG * $-\boldsymbol{a t i}>\mathrm{Ka}-\boldsymbol{n}:-\boldsymbol{a d i}$ 'ver', Ab -atfi-pi, Mo -atfaGan, ToAnt <atianivá>, To -atf-aGan 'mostrar'.

M: PM1 * $\boldsymbol{a} \boldsymbol{t}$ > Mk - $\boldsymbol{e}$ - $\boldsymbol{h i n}$-ix 'mostrar (algo) a alguien', Ni -t'-at 'mirar', 'prestar atención'

(11) G: $\mathrm{PG}{ }^{*}$-at'ó > Ka -ato:, Mo -ato:, Pi -ató-tak, To -ato-tak 'bostezar'.

M: PM1 *at'o > Mk -ot'o-kii, Ni - t'-at'o-?in 'bostezar'.

(12) G: PG *-awi?a > Ka -awi:, To -awa: 'cazar'.

$\mathrm{M}: \mathrm{PM}{ }^{*}$-wo?j > Ma -wo?oj, $\mathrm{Ni}-\boldsymbol{w o}$ ?j, Cho -woxi?, Wu -wojin 'buscar'11.

(13) G: PG * -eké?e > Ka -igi-wa 'comer con', Mo -eke?e, Pi -eké?e, To -eke?e 'comer'.

$\mathrm{M}: \mathrm{PM}{ }^{*}-\boldsymbol{e} \boldsymbol{k}>\mathrm{Mk}-\boldsymbol{e k}$, Cho -jekju?, Wi -?ek 'comer'.

(14) G: $\mathrm{PG}{ }^{*}$-eko $\sim{ }^{*}$-iko > Ka -igo, $\mathrm{Ab}$-ik -eek, Mo -ik -e:k, Pi -hek -?ek, To -ik $\sim$-hek 'irse'.

$\mathrm{M}: \mathrm{PM}{ }^{*}-\mathbf{\Lambda k} \sim{ }^{*}$-ek $\sim{ }^{*}$-ik $>\mathrm{Mk}-\mathbf{k} \sim-\mathbf{u k}, \mathrm{Ni}-\mathbf{\Lambda} \mathbf{k} \sim-\mathbf{i t} \int$, Cho -ak-i $\sim$-ek-i, Wi -ek $\sim$-ik 'irse'.

(15) G: $\mathrm{PG}{ }^{*}$-elot'é ${ }^{*}$-elot'í > Ka -jo:te, Ab -aatf(i)- -oatf- -aate -oate, Pi -otfé, To $-(\boldsymbol{o})$ ?ot $\boldsymbol{i} \boldsymbol{i}$ 'dormir'.

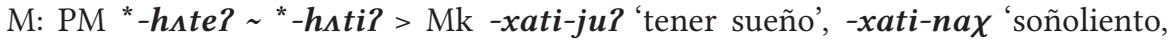
dormilón', Ni n-xate 'sueño', Cho ixnjeti? (< “in-xeti?) 'sueño', Wi na-hate 'sueño'.

(16) G: PG *-i?áts'-en > Ka -iwatfin-aGan 'tener una idea', -iwatfin-aGan-Gatidi 'educar', Pi -jjáten, To -jaten 'saber, entender'

$\mathrm{M}: \mathrm{PM}$ *-ejhats-han > Mk -ejxatshen, $\mathrm{Ni}$-ejxatsxan, Cho -ejesan 'saber'.

G: $\mathrm{PQ}$ *-kef'óqo > Mb <-gechogo> "picotear el paxaro", Ab -akehaR-hek

'cortar palo', Pi -kesóq 'picar como leña', 'hachar', To -kesoq 'hachar'.

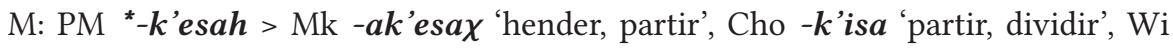
-t $\int$ esah 'hender, partir'.

(18) G: PG * $\boldsymbol{m o}>$ Ka $\boldsymbol{e} \boldsymbol{m}$-i, Mo $\boldsymbol{m o}$-?e?, Pi ha-mó '¡vete!'12.

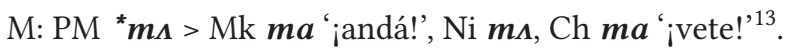

11 En algunos diccionarios de lenguas mataguayas, estos verbos aparecen a veces glosados como 'cazar', por ejemplo en maká (Belaieff 1940:49), en niwaklé (Stell 2000), etc.

12 En Ka se trata de la segunda persona supletiva del verbo de la serie (14) no solo en imperativo sino también en el modo indicativo ('vas'), en Mo y $\mathrm{Pi}$-mo- aparece en la segunda persona singular de imperativo. Para el PQ se puede reconstruir una forma de

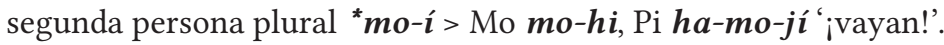

13 En Ni y Cho esta forma es la segunda persona supletiva utilizada para el imperativo del verbo 'ir' de la serie (14); la forma Mk no es considerada un verbo sino una "partícula" por Gerzenstein (1999). Recientemente, he sugerido que el prefijo Mk $\boldsymbol{m} V$ - ' 2 a . p. sing., noindicativo’ también provendría de PM “ms ‘ivete!’ (Viegas Barros 2012). 
(19) G: $\mathrm{PPT}{ }^{*}$-n-áto > Pi -náto, To -nato 'reunir, recoger'.

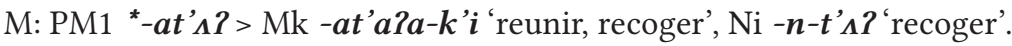

(20) G: PG * ${ }^{\boldsymbol{n}} \boldsymbol{n}$-ij'ó 'lavarse' > Ka $\boldsymbol{n}$-j:o-God 'agua', Pi -n-ijó, To -n-jo 'lavarse', $\boldsymbol{n}$-joGot 'agua para lavarse'.

M: PM *-naj 'bañarse' > Ni - $\boldsymbol{n} \boldsymbol{a j}$ 'bañarse', -naj-xat 'agua para bañarse', Cho - $\boldsymbol{n} \boldsymbol{a j} \boldsymbol{j}$ njate, Wi -naj 'bañarse`.

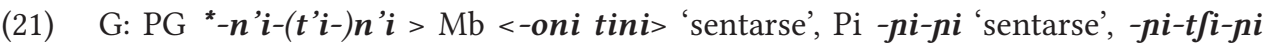
'estar sentado', 'sentarse ${ }^{\prime 14}$.

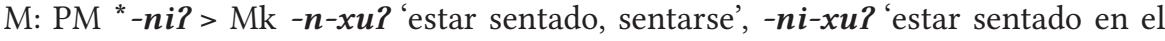
suelo', Cho -ine? 'sentarse',Wi - $\boldsymbol{n i}$ 'sentado, quieto' (demostrativo)

(22) G: PG ${ }^{*}$-oma > Ka -o:ma-te-wa 'acostarse juntos', Mo -om-ala?, To -m-ala? 'cama'.

$\mathrm{M}: \mathrm{PM}^{*}-\boldsymbol{m a} \boldsymbol{2}>\mathrm{Mk}-\boldsymbol{m a} \mathbf{2}, \mathrm{Ni}-\boldsymbol{m a}$, Cho -maa, Wi - $\boldsymbol{m a} \boldsymbol{a}$ 'dormir'.

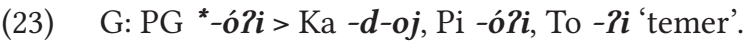

M: $\mathrm{PM}{ }^{*}-(\boldsymbol{w}) \boldsymbol{\Lambda} \boldsymbol{j}>\mathrm{Mk}$ (Belaieff) $<-\boldsymbol{a} \boldsymbol{j}>$ 'temer', $\mathrm{Ni}-\boldsymbol{\Lambda} \mathbf{\imath} \boldsymbol{j} \sim-\boldsymbol{\Lambda} \boldsymbol{j}$ 'estar preocupado, estar triste, ser respetuoso', Wi - waj 'temer'.

(24) G: PGS *-o?wo > Ab -eoe, -eoa- 'estar', Mo ?we, Pi ?wo 'hay', 'existe', To -o?o 'estar presente'.

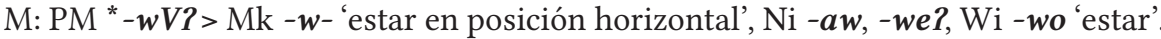

(25) G: PG * -t'ap'élV > Mb <-topile> 'atascarse la comida', Mo -(a)tapla, Pi -(i)tapéla, To -tapla 'atragantarse'.

M: PM *-op'ale > Ni -op'akle 'atragantarse', Cho -op'ali?n 'tener hipo', Wi -ople 'hipar'.

(26) G: $\mathrm{PG}{ }^{*}$-walek > Ka -waligi 'andar', Pi -walek-ta-lége 'arrastrarse', To-walek-ta 'caminar despacio'.

$\mathrm{M}: \mathrm{PM}{ }^{*}$-welek > Mk -iwelki-met 'cojo'15, $\mathrm{Ni}$-wakletf 'caminar, andar', Cho -wilik 'irse al monte', Wi -welek 'viajar'.

(27) G: PG *-wen > Ka -win 'mirar', Mb <-guiini > 'mirar, ver', Ab -eon, $\mathrm{Pi}$-wan-á 'encontrar', To -(a)wan-a 'ver'.

$\mathrm{M}: \mathrm{PM}^{*}$-wen > Mk -wen 'ver', Ni -wan 'ver, encontrar, conocer', Cho -ween 'ver, encontrar', Wi -wen 'ver'.

(28) G: PG *-Pelote > Ka -elodi 'correr rápido, huyendo', Pi -elot-âakí 'huida', To - elt $-\boldsymbol{a G a j - k}$ 'huidor'.

M: $\mathrm{PM}{ }^{*}-i l_{\Lambda} t>\mathrm{Mk}$-ilat, $\mathrm{Ni}-\boldsymbol{k} \boldsymbol{l}_{\Lambda} t$, Cho -jilt-an 'huir'.

14 La sílaba * $\boldsymbol{t}^{\prime} \boldsymbol{i}$ - es epentética, como lo es también en la serie (32). La raiz se encuentra

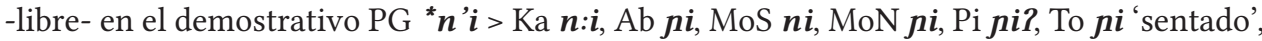
y gramaticalizada en el sufijo direccional $\mathrm{PG}^{*}{ }^{-} \boldsymbol{n} \boldsymbol{i}>\mathrm{Ka}-\boldsymbol{n}:(\boldsymbol{i}),>$ Ab -api $\sim-\boldsymbol{i} \boldsymbol{n} \mathbf{i}, \mathrm{MoN}-(\boldsymbol{i})$ $\boldsymbol{j i}$, $\mathrm{Pi}-(\boldsymbol{i}) \boldsymbol{j i}$, To -(i) $\boldsymbol{n i}$ 'hacia abajo'.

15 Sincrónicamente, -met no parece funcionar como un sufijo en Mk; pero cf. el sufijo Ni -mat, formador de adjetivos referidos a defectos físicos. 
(29) G: PG * -1em > Ka -ee:m 'morir', Ab -aham-Raik 'muerto', Mo -1o:m, Pi - To:m, To - $\mathbf{0}: \boldsymbol{m}$ 'apagado'.

M: PM *-om > Mk -om, Cho -om, Wi -j-om 'apagarse'.

$(30)^{16}$ G: $\mathrm{PG}{ }^{*}$-Pem-ate > Ka -ee:madi, Ab -ahamat 'matar', Mo -om-at 'terminar, apagar', Pi -mat, To -(o)mat 'terminar'.

$\mathrm{M}: \mathrm{PM}$ *om-hate ${ }^{*}$-om-ate > Mk -omhet, Cho -omate?, Wi -omet 'apagar'.

(31) G: PG *-7ot'án > Ka -otan, Ab -haten, Pi -Potán, To -?otan 'despreciar'

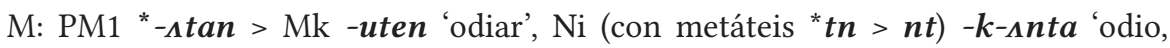

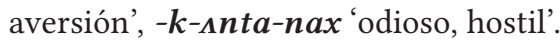

(32) G: PG *-Potsi-t'i-n'í > Ka -odzitini 'caer (un inanimado)', Pi -Potfitfiní 'caerse'.

M: PM1 ${ }^{*}-\boldsymbol{\Lambda} t \boldsymbol{s i}>\mathrm{Mk}-\boldsymbol{a t s i}$-, Ni $-\boldsymbol{\Lambda} t \boldsymbol{t} \boldsymbol{i}$ 'derramar'.

Cf. también (87), (88), (92), (133).

\section{Cualidades y estados}

(33) G: PG *-alem 'estar calvo' > Ka -ale 'corona', Ab -(h)alem 'estar calvo', -alem-Ra 'parte afeitada de la cabeza'.

M: PM1 *leme $(\boldsymbol{m})$ > Mk leeme (plural lemem) 'liso', Ni klamam 'liso, despejado, limpio, 17 .

(34) G: PG * $-\boldsymbol{e} \boldsymbol{j} \boldsymbol{i}>\mathrm{Ka}-\boldsymbol{e} \boldsymbol{j}$ 'tener fruto', Ab - $\boldsymbol{e} \boldsymbol{i}, \mathrm{Mo}-\boldsymbol{j} \boldsymbol{i}, \mathrm{Pi}-\boldsymbol{j} \boldsymbol{i}$, To - $\boldsymbol{j} \boldsymbol{i}$ 'estar maduro'.

$\mathrm{M}: \mathrm{PM}{ }^{*}-\mathrm{ju}$ ?.> Ni -ji?j, Cho -jowe?, Wi -ju? 'maduro'.

(35) G: PG *-ewela (o *-ewel'a) > Mb <-eguila > 'cansarse', Ab -aal 'ser lento', 'tener pereza, cansancio', Mo -(e)wal, Pi -(o)wál 'ser perezoso, haragán', To -wal 'ser perezoso, haragán'.

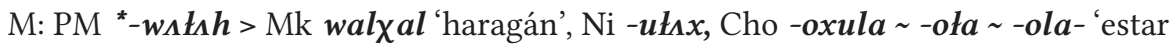
cansado', Wi -iwal 'ser lento'.

(36) G: $\mathrm{PG}{ }^{*}$-iweq'a- > Ka -iwaqa-paGadze-gi 'arruga', Mb <-iguacape > 'arrugarse', To -iwigaGa?t 'arrugado'.

M: PM ${ }^{*}-\boldsymbol{u} \boldsymbol{w} \boldsymbol{q}$ ' $\boldsymbol{h} \boldsymbol{h}>\mathrm{Mk}-\boldsymbol{o} \boldsymbol{w q}$ 'o-tsi? 'arruga', Ni -ukax 'hinchazón', Wi (dialecto bazanero) $-\boldsymbol{t}-\boldsymbol{i} \boldsymbol{k}^{w} \boldsymbol{a}$ 'hinchazón', (dialecto weenhayek) $-\boldsymbol{t}-\boldsymbol{u} \boldsymbol{k}^{w} \boldsymbol{a}$ 'hinchar'.

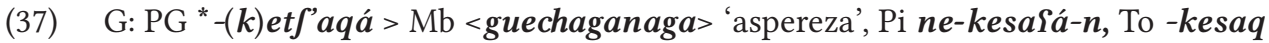
'áspero'.

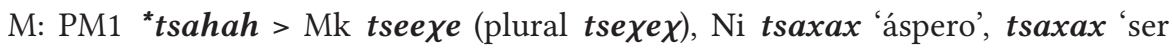
áspero'.

16 En ambas familias, la raíz es la misma que la de la serie (29), seguida en este caso del sufijo de la serie (126).

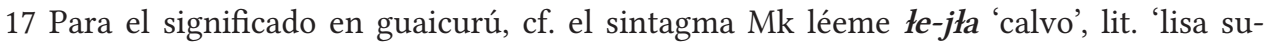
cabeza'. 
(38) G: PG * ${ }^{*} \boldsymbol{o t}$ 'j-áq $\boldsymbol{a}>\mathrm{Mb}<-\boldsymbol{o t i g} \boldsymbol{a}>$ 'estar gordo', <eotiga $>$ 'crasso, gordo', MoN -otfaq, ToAnt <yotiac> "gordo está", <yutiach> 'gordo, grueso', To -otfaq 'gordo'. M: PM *-ıtah > Ni - 1 tex, Cho -atax, Wi -j-atah '(ser) gordo'.

(39) G: PG *owadjá? > Ka owa:di, Ab aoari, Mo wa:dza?, Pi wadjá-\{aj-k, To waza? 'mellizos, gemelos'.

M: PM *wetshV-> Mk wetsxuk 'dos', wetshet-k'-ewil 'tres' (lit. 'dos-y-uno'), Cho iwje?tsjela 'tres'.

(40) G: PPT *qapí > Pi qapí, To qapi `pequeño', -qapi-aqtfit 'achicar’.

M: PM1 *qapap > Mk qepepep, Ni kapap 'enano'18.

(41) G: PG * $q$ 'owa- > Ka do-qoa-Ga, To qow-aGan-aG-aj-k 'débil'.

M: PM * ${ }^{*} \boldsymbol{a} \boldsymbol{a} \boldsymbol{h}^{w} V(-\boldsymbol{n})>\mathrm{Mk}-\boldsymbol{q} \boldsymbol{e w x u}$ ?'muy flaco', Ni - $\boldsymbol{k a f u n}, \mathrm{Wi}-\boldsymbol{k e h}^{w} \boldsymbol{i}(\boldsymbol{n})$ 'ser débil'.

(42) G: PQ * Tim > Mo Rim 'está seco', Pi Rim Rem, To Rim Rem 'seco'.

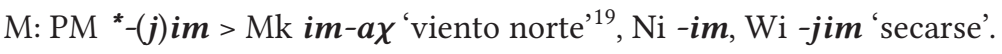

Cf. también (86), (95), (132).

\section{Partes y funciones del cuerpo humano y animal}

(43) G: PG * ${ }^{*} \boldsymbol{a d}$ 'e $>$ Ka - $\boldsymbol{a}: \boldsymbol{d e}, \mathrm{Ab}-\boldsymbol{a r}(\boldsymbol{e}), \mathrm{Mo}-\boldsymbol{a d e}$ 'pestaña'.

M: PM *tsi? *-si? 'pelos de las pestañas y cejas', en *tו ${ }^{*}$-tsi? > Ma -tetsi?,

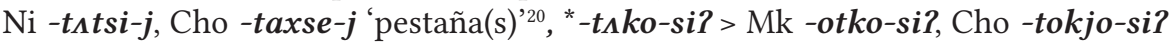
'ceja(s)' ${ }^{21}$.

(44) G: $\mathrm{PG}{ }^{*}$-ajaqats'V > Ka -wajaGatfi 'artejo', Pi -ja\{atá, To -jaGa:ta 'dedo de la mano'.

M: PM *-jaqsi? > Mk -jaqsi?, Cho -jesiki?'dedo'.

(45) G: PG *-(a)pijó > Ka -bjo-go, Pi -apijó, T -pijo 'grasa'.

$\mathrm{M}: \mathrm{PM}^{*}$-ape? > Ni -ape?, $\mathrm{Ch}-a p e ?, \mathrm{Wi}-k$-ape?'aceite'.

(46) G: $\mathrm{PG}{ }^{*}$-at'o $>\mathrm{Mb}<-\boldsymbol{a t o l o}>$ 'coronilla de la cabeza', Mo r-oto 'coronilla', -oto-senta 'llevar sobre la cabeza'.

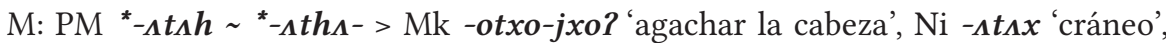
Cho (dialecto yowuwa) $-\boldsymbol{a} \boldsymbol{w} \boldsymbol{t a} \boldsymbol{-} \boldsymbol{k}$ 'vincha'.

18 En mataguayo, aparentemente, con reduplicación parcial de la raíz, como en (33); en Mk - en este caso - con triplicación parcial. Mk qepepep se aplica a seres animados en general, Ni kapap solamente a perros.

19 Etimológicamente: *'el [viento] seco'.

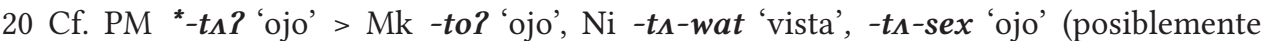
también Cho -tatex 'ojo').

$21 \mathrm{Cf} . \mathrm{PM}{ }^{*}-t_{\boldsymbol{\Lambda}} \boldsymbol{k o}>\mathrm{Mk}$-otko-jek -itko-jek 'frente', Ni -tı?ko 'cara', 'frente', Cho -tokjo 'cara', Wi -tıtfo 'frente'. 
(47) G: PGS *-awel > Ab -aoel 'entrañas', 'corazón', Mo awele-sek, Pi -ajl-sik 'tripa', To -awel 'barriga'.

M: $\mathrm{PM}{ }^{*}-w e l$ > Ni -wa?k (plural -wakl-aj) 'intestino', Cho -ele 'tripa'.

(48) G: $\mathrm{PG}{ }^{*}-\boldsymbol{a} \boldsymbol{w}^{\prime} \boldsymbol{a} \boldsymbol{~ > ~ K a ~ - a b : a - d i , ~} \mathrm{Ab}-\boldsymbol{a o a}$, Mo - $\boldsymbol{a} \boldsymbol{w a} \boldsymbol{a}, \mathrm{Pi}-\boldsymbol{a} \boldsymbol{w a ́}$, To - $\boldsymbol{a} \boldsymbol{w a} \boldsymbol{a}$ 'ala'.

M: PM * $\boldsymbol{t}-\boldsymbol{a} \boldsymbol{h}^{\boldsymbol{w}}>$ Mk tef, Ni laf, Cho tax, Wi teh ' 'ala'.

G: $\mathrm{PG}{ }^{*}-\boldsymbol{e l}$ 'aq $\boldsymbol{a}>\mathrm{Ka}-\boldsymbol{e l}: \boldsymbol{a G a}$, Mo -(e)laq, To -laq 'espalda'.

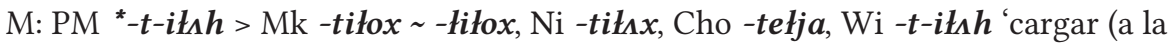
espalda)'22.

(50) G: PG * -w'it'i > Mb <-buite > 'peerse', <-buite-ga $>$, Ab -oitfi 'pedo'.

M: PM * ${ }^{*} \boldsymbol{h}^{w} \boldsymbol{u t u}$ ? > Mk -eftu? -iftu?, Ni -fu?t, -ftu-t, Wi (dial. bazanero) $\boldsymbol{t}^{\prime}-\boldsymbol{e} \boldsymbol{h}^{w} \boldsymbol{t}$ $\boldsymbol{e j}$ 'pedo(s)'.

(51) G: PG ${ }^{*}-\boldsymbol{k}^{\prime} \boldsymbol{e} \boldsymbol{t}^{\prime} \boldsymbol{e}-\boldsymbol{l} \boldsymbol{V} \sim{ }^{*}-\boldsymbol{t}^{\prime} \boldsymbol{e} \boldsymbol{k}^{\prime} \boldsymbol{e} \boldsymbol{e}-\boldsymbol{l} \boldsymbol{V}^{23}>\mathrm{Mb}<-\boldsymbol{a t i q u i l o}>$ 'cera... de las orejas' ${ }^{24}, \mathrm{Ab}$ -ketal, Mo -kePla, Pi -ketéla, To -tela 'oreja'.

M: PM2 * $\boldsymbol{k}^{\prime}$ 'ote > Cho -kjote?, Wi $-\boldsymbol{t}$ '’ote (dialecto weenhayek $-\boldsymbol{k}^{j}$ 'ote?) 'oreja'.

(52) G: PQ * $-\boldsymbol{o j i k}>\mathrm{Mo}-\boldsymbol{o j k}$, $\mathrm{Pi}-\boldsymbol{o j k}$, To $-\boldsymbol{o j} \boldsymbol{k}$ 'derecha'.

M: $\mathrm{PM}{ }^{*}-\boldsymbol{h}^{w} \boldsymbol{e j i h}>\mathrm{Mk}-\boldsymbol{f} \boldsymbol{e j i x}$ 'izquierda', Ni -fajif, Cho $-\boldsymbol{f}^{w} \boldsymbol{e j e}$ 'derecha'.

(53) G: PG * -qon'á > Ka -Gon:a-gi 'pie, pierna', Pi -qoná, To -qona 'dedo del pie'.

M: PM *-qınah > Mk -qanaxak 'pierna', Cho -akoxna 'muslo', 'pata'.

(54) G: $\mathrm{PG}{ }^{*}-\boldsymbol{q} \boldsymbol{o t}$ 'e $>\mathrm{Mb}<-\boldsymbol{o g o t e}>$ 'paladar', To $\boldsymbol{l}$-mik $\boldsymbol{l}$-qote 'hueso nasal' ${ }^{25}$.

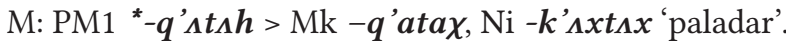

(55) G: $\mathrm{PG}{ }^{*}-\mathbf{p} \boldsymbol{a} \boldsymbol{k o}>\mathrm{Ka}$-wako-go, $\mathrm{Ab}-\boldsymbol{a k}$, $\mathrm{Pi}$-ó?ok, To - $\mathbf{p o k}$ 'cuero'.

$\mathrm{M}: \mathrm{PM}{ }^{*}-\boldsymbol{\Lambda} \boldsymbol{h}>\mathrm{Mk}-\boldsymbol{a x}, \mathrm{Ni}-\boldsymbol{\Lambda} \boldsymbol{x}$, Cho $-\boldsymbol{a x}$, Wi $-\boldsymbol{\Lambda} \boldsymbol{x}$ 'cuero, piel'.

(56) G: PG *-1okel *-Polek > Ka -okel-igi 'lengua', Ab-eelg- 'lamer', Mo-o1leg-GanaGat 'lengua', Pi -lolek 'lamer'.

M: PM *-kahlik'u > Ni -tfaPletf, Wi -kahlitf'u 'lengua'.

G: PGS *-Potá > Ab -etata ${ }^{26}$, Mo -?o:ta, Pi -Potá, To -Pota 'vena'.

$\mathrm{M}: \mathrm{PM}^{*}-\boldsymbol{\Lambda} \boldsymbol{t}$ '> Mk - ath-ii 'menstruar', -ath-its 'sangre', Ni - $\boldsymbol{n}-\boldsymbol{\Lambda} \boldsymbol{t}$, Cho -at'-ni 'sangrar'. Cf. también (91).

22 La segmentación del prefijo $\boldsymbol{t}$ - en esta forma Wi fue establecida por Vidal (2011:149). 23 Sin el sufijo *-lV, cf. PQ *-kéte > Mo - kete 'patilla', Pi kéte-k 'disco auricular (adorno)', To - ket 'asa, manija'.

24 En Ka esta forma se conservó en el derivado -atikilo-naGa 'sordo'.

25 Etimológicamente *'paladar de la nariz'.

26 Con reduplicación y disimilación vocálica de pre-Ab * ${ }^{*}$ atata. Una reconstrucción distinta en Viegas Barros (2013: 259, serie 684). 


\section{Partes de plantas}

(58) G: $\mathrm{PG}{ }^{*}-\boldsymbol{a} \boldsymbol{w o}-\boldsymbol{q} \boldsymbol{o}^{27}>\mathrm{Ka}-\boldsymbol{a} \boldsymbol{w o G o}, \mathrm{Pi}-\boldsymbol{a}$ ?wó, To -awoGo 'flor'.

$\mathrm{M}: \mathrm{PM}^{*}-\Lambda$ wo $>\mathrm{Ni}-\Lambda$ wo, Cho kawo lawo, Wi ławo 'flor'.

(59) G: PG *el'-á > Ka el:a, Mo la, Pi halá, To ala 'fruta'28.

M: $\mathrm{PM}^{*}-\boldsymbol{a}$ ? > Mk $\boldsymbol{1}-\boldsymbol{e}$ ?, Ni $-\boldsymbol{a}$ ?, Cho $\boldsymbol{1}-\boldsymbol{a} \boldsymbol{a} \boldsymbol{a}$, Wi $\boldsymbol{t}-\boldsymbol{a}-\boldsymbol{j}$ 'fruta'.

(60) G: PGS *-petá > Ab -peta 'grano, fruta, semilla', Pi -petá 'semilla'.

M: PM *-pıta? > Ni -n-pıta, -pıt-xan, Cho -pa?te?'desgranar'.

Cf. también (90).

\section{Flora y fauna}

(61) $\mathrm{G}: \mathrm{PG}^{*}-(\boldsymbol{k})$ etf'eqa $>\mathrm{Mb}<$ guechegega $>$, Ab oeheg-Ra, To -kesaGa 'sarna'.

M: $\mathrm{PM}{ }^{*}-\boldsymbol{k} \boldsymbol{e t s}$ 'Vh > Mk -exkits'i 'sarna de cabeza', Cho ti-kiso? 'sarnoso', Wi $\boldsymbol{t} \boldsymbol{e} \boldsymbol{e s} \boldsymbol{a h}$ (dialecto weenhayek $\boldsymbol{k}^{j} \boldsymbol{e s a}$ ) 'sarna'.

(62) G: PPT *májo > Pi májo? 'pájaro, ave', To majo 'ave grande'.

M: PM * $\boldsymbol{m} \boldsymbol{i j o}>$ > Mk $\boldsymbol{m} \boldsymbol{i j o}$ 'águila', Ni mijo 'especie de gavilán', Cho $\boldsymbol{m i j o - k i}$, Wi mijo 'águila, aguilucho (Busarellus nigricollis)'.

(63) G: PG *tsjáw- > Ka edziwa, MoS (Paucke) <atiavic $>$, MoN t $\int \boldsymbol{a}: \boldsymbol{w i k}$, Pi $t$ tajk, ToAnt < tiabich $>$ ('palma'), To t $\int a j k$ 'palmera (Copernicia alba)'.

M: PM ${ }^{*} \boldsymbol{h}^{\boldsymbol{w}} \boldsymbol{i t s}-\boldsymbol{u} \boldsymbol{k}>\mathrm{Mk}$ fitsuk, Ni ftsu?k, Cho wisjuk, Wi $\boldsymbol{x}^{\boldsymbol{w}} \boldsymbol{i t s u k}$ 'palmera (Copernicia alba)'.

Cf. también (65) y (85).

\section{Naturaleza no animada}

(64) G: PG * ${ }^{*} \boldsymbol{a j i} \mathbf{i}$ > Ka -waj, Ab -oii, Mo - ahi, Pi -aí, To -aji 'lado'.

$\mathrm{M}: \mathrm{PM}^{*}-\left(\boldsymbol{h}^{w}\right) \boldsymbol{a j} \boldsymbol{i}>\mathrm{Mk}-\boldsymbol{w e j}$ 'estar en posición lateral ${ }^{29}, \mathrm{Wi}-\boldsymbol{x}^{w}$ aje 'estar al costado, a la orilla', -aje 'compañero', Cho - aji 'compañero de camino'.

27 Derivado de la raíz conservada en PPT *-awó > Pi -awó, To -awo 'florecer'.

28 En guaicurú la protoforma era originariamente ${ }^{*}-\boldsymbol{a}$ 'fruta', gramaticalizada en el sufijo clasificador nominal $\mathrm{PG}{ }^{*}-\boldsymbol{a}>\mathrm{Ka}-\boldsymbol{a}$, To $-\boldsymbol{a}$ 'fruta'. El sustantivo se alargó por incorporación del alomorfo del prefijo de tercera persona poseedora * $\boldsymbol{e l}$ '- de la serie (102), posiblemente debido a la tendencia universal a alargar monosílabos originarios. Nótese que un alargamiento paralelo ha ocurrido, independientemente, en la mayoría de las lenguas mataguayas.

29 También 'estar en posición ántero-posterior'. 
(65) G: PG *alVwa (o *al'Vwa) > Ka apil-alo ${ }^{30}$, Pi aléwa 'tacurú, nido de termitas'31. M: PM *huwa > Ni huwa, Wi huwa 'hormiguero'.

(66) G: $\mathrm{PG}$ *alo > Mb <-alogeni> 'poner ceniciento, encenizar', Mo alo, To alo 'ceniza'. M: PM * lo? > Mk lo? 'ceniza', Cho -lo? 'resto quemado, ceniza'.

(67) G: PG * -am'oqo > Ka -am:oGo, Ab-agma-jaRa, Mo -amoGo-jaGa, To -amoGojaGa 'polvo'.

$\mathrm{M}: \mathrm{PM}{ }^{*}-\boldsymbol{m} \boldsymbol{\Lambda} \boldsymbol{q}$ ' > Ni - $\boldsymbol{m} \boldsymbol{\Lambda} \mathbf{k}$, Wi $\boldsymbol{m o k}$ ' 'polvo'; Cho - $\boldsymbol{m a k}$ 'harina'.

(68) G: PG *-(a)niko> Ka -anigo 'olor', Ab n(i)k-at-RanRat 'nariz', Mo pik-saq

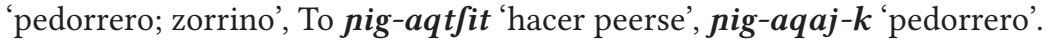

M: $\mathrm{PM}^{*}-(\boldsymbol{a}) \boldsymbol{n i h}>\mathrm{Mk}-\boldsymbol{e n j i x}, \mathrm{Ni}-\boldsymbol{n i}$ ?\}, Cho -ni (dialecto yowuwa -ne), Wi -nih 'olor'.

(69) G: PG *aqati > Mb < agati-ye $>32$ 'lucero de la tarde', MoN waqahni, Pi waqtfiní, ToAnt <avacatini>, To waqa:ni 'estrella'33.

M: PM ${ }^{*}$ qate-ts > Ni kati?s, Cho kates, Wi kates (pl. katets-el, dialecto weenhayek qates) 'estrella'.

(70) G: PG * -atfék > Mb <-ayaqueni> 'labrar madera', Ab -ahak 'palo, tabla', -ahak 'labrar (madera)', Pi - asék, To - $\boldsymbol{a} \boldsymbol{j} \boldsymbol{i} \boldsymbol{k}$ 'leña'.

M: PM1 * $\boldsymbol{a h} \boldsymbol{a} \boldsymbol{k}(\boldsymbol{V})>\mathrm{Mk} \boldsymbol{n}$ - $\boldsymbol{a x} \boldsymbol{a} \boldsymbol{k}$ 'leña, madera, palo, vara', $\boldsymbol{n}$-axka-k 'árbol', Ni $a ? k x i-j u k$ (metátesis de *a?xki-) 'árbol'.

(71) G: PG * ${ }^{*} \boldsymbol{a} \boldsymbol{w}^{\prime} \mathbf{e} g$ '> Ka -b:e:g:i, Mo -awak, Pi -awák, To -wak 'agujero'.

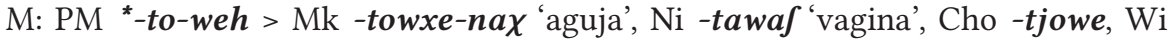
-toweh 'agujero'.

(72) G: PG *inaqá > Ka nika-Ga-pi 'año', Ab iniega-, Mo pa:Ga 'verano, año'.

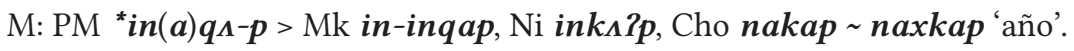

(73) G: PG *-ol'ét > Ka -ol:edi, Mo -ole? -olit-, Pi -olé? 'fuego', To -le? 'fósforos, tizón, fuego'.

M: PM *-VletV? > Mk -litu? 'leño semiquemado', Cho -ałet 'leña encendida', Wi -let 'leña'.

30 Ka apil- ipil- 'termita o insecto similar', cf. ipil-aGawa:-na 'termita'.

31 La forma Pi parece estar remodelada analógicamente sobre la base de PG * Paléwa 'tierra', cf. la serie (75). También pertenece a esta serie MoS (Tavolini) < callegá > "especie de hormiguero", que representa gráficamente con $<\boldsymbol{g}>$ a la consonante $/ \boldsymbol{w} /$.

32 En GN se esperaría * $\boldsymbol{a}$ Gadi- (Mb * $<\boldsymbol{a g} \boldsymbol{a d i}$-ye $>$ ), pero el sufijo GN - $\boldsymbol{d} \boldsymbol{z} \boldsymbol{e}$ puede provocar el ensordecimeinto de la última consonante sonora precedente, cf. Ka ba:Gate-dze 'anillo' de ba:Gadi 'mano', nigotaqa-dze-gi 'ciudadano' de nigotaGa 'ciudad', etc.

33 Las formas Mo, Pi y To reflejan PQ *aw-aqati-ni, con incorporación de PG *áwi (> Ka awi: 'tarde', Mo awit wit lawit, Pi háwit, To hawit 'atardecer, tarde'), más el sufijo direccional PG ${ }^{*}-\boldsymbol{n}$ 'i 'hacia abajo', véase la nota 14. 
(74) G: PG * qalat'i > Ka Galati, Ab akalatf, Mo qalatfi, To latfi 'granizo'.

M: PM *qalatu? *halatu? > Ni xala?tu, Cho alatju?, Wi kalatu (dialecto weenhayek qalatu?) 'granizo'.

(75) G: PGS * ?al'éwa > Ka ap-al:wa-Ga, Ab aaloa, Mo Plawa, Pi Paléwa, To Ralwa 'tierra'.

M: PM *ahu > Ni $\boldsymbol{n}$-ahu 'tierra', 'mundo', Wi alo-tah 'campo', ało-ha 'afuera'.

(76) G: PG *?ewjá > Ka ewi:-di, Ab aoi, Mo ?ja?, Pi ha?já, To Paja 'rocío'.

M: PM *ija-ts > Mk ijets, Ni jija?s, Cho -jis-ta, Wi ijas 'rocío'.

Cf. también (94), (134).

\section{Relaciones familiares y sociales}

(77) G: PG ${ }^{*}-\boldsymbol{e t f} \boldsymbol{i}-{ }^{*}{ }^{*}-\boldsymbol{e t \int} \boldsymbol{j}->\mathrm{Ka}-\boldsymbol{e t f i - d z e , ~ M b ~ < e i c h i y o > ~ ' h u e ́ r f a n a ' , ~ T o ~ - a s o - \int i ~}$ 'hijastra', 'sobrina'.

$\mathrm{M}: \mathrm{PM}{ }^{*}-\boldsymbol{\Lambda} \boldsymbol{s}>\mathrm{Mk}-\boldsymbol{a s}$, Cho - as, Wi -ths 'hijo'; $\mathrm{PM}{ }^{*}-\boldsymbol{\Lambda} \boldsymbol{s}-\boldsymbol{e}$ ? > Mk $-\boldsymbol{a s i}, \mathrm{Ni}-\boldsymbol{\Lambda}$ ?se?, Cho -axse, Wi -łnse? 'hija'.

(78) G: PGS *-jalé > Ab -ioale, Mo -jale, Pi -jalé, To -jale 'hija'; PGS *-jalé-k $>\mathrm{Ab}$ -ioalek, Mo -jalek, Pi -jalék, To -jalek 'hijo'.

M: PM *-le-ts > Mk -lits, Cho -les, Wi -les 'conjunto de hijos, familia'.

(79) G: PPT *koté > Pi koté, To kote 'abuela'.

M: $\mathrm{PM}{ }^{*}-\boldsymbol{k} \boldsymbol{\Lambda} \boldsymbol{t}>\mathrm{Mk}-(\boldsymbol{e w}) \boldsymbol{k e t}$ 'abuelo', $-(\boldsymbol{e w}) \boldsymbol{k e t}-\boldsymbol{i}$ 'abuela', Wi $-\boldsymbol{t} \int \boldsymbol{a t i}$ (dialecto weenhayek $\left.-\boldsymbol{k}^{j} \boldsymbol{a t} \boldsymbol{i}\right)$ 'abuelo'.

Cf. también (93).

\section{Pronombres personales}

(80) G: PG *ej-ém' > Ka e:m: e:, Ab aim, Mo ajim jim, Pi hajém, To ajim 'yo'. M: PM2 ${ }^{*} \boldsymbol{j}$-am > Cho ja?am, (dialectos yowuwa y manjuy) jem, Wi (dialecto weenhayek, sociolecto "familiar”) jam 'yo'.

(81) G: PG * $\mathbf{R} \boldsymbol{a m}$ '> Ka (sociolectos noble y femenino) $\boldsymbol{a} \boldsymbol{m}:-\boldsymbol{i}, \mathrm{Mb}$ (habla femenina) $<\boldsymbol{a m}$, $a m i>$, Pi Ram, To Pam qam 'tú'34.

M: PM2 * am > Cho a?am, (dialectos yowuwa y manjuy) am, Wi am 'tú'.

(82) G: PGS *al-am > Ab halam 'le, lo', 'lo de él', Mo alam, ToAnt < halám> 'él', 'ella'. M: PM2 1-am > Cho tam, Wi tam 'él, ella'

$34 \mathrm{La}$ variante con $\boldsymbol{q}$ - inicial en To posiblemente por influencia del pronombre de $2^{\mathrm{a}}$ persona plural qami 'uestedes'. Lo mismo habría ocurrido en los pronombres de $2^{\mathrm{a}}$. Persona singular del Ab (akami) y del Mo (qami?). 


\section{Demostrativos}

(83) G: PG *n'a > Ka $\boldsymbol{n}: \boldsymbol{a}$, Ab - $\boldsymbol{n} \boldsymbol{a}$, Mo $\boldsymbol{n} \boldsymbol{a}$, Pi $\boldsymbol{n} \boldsymbol{a}$ ? no?, To $\boldsymbol{n} \boldsymbol{a}$ 'próximo, en movimiento'.

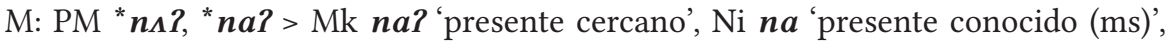
$\boldsymbol{n \Lambda}, \boldsymbol{n} \boldsymbol{a}$ - 'demostrativo de proximidad', Cho $\boldsymbol{n} \boldsymbol{a} \sim \boldsymbol{n \boldsymbol { i }}$ 'cercano', Wi $\boldsymbol{- n \boldsymbol { a }}$ 'demostrativo posicional de distancia mínima'.

(84) G: PG * $\boldsymbol{k}^{\prime} \boldsymbol{a}>\mathrm{Ka} \boldsymbol{k} \boldsymbol{a}$, Ab - $\boldsymbol{k} \boldsymbol{a}$, Mo $\boldsymbol{k} \boldsymbol{a}$, Pi $\boldsymbol{g} \boldsymbol{a}$ ?, To $\boldsymbol{k} \boldsymbol{a}$ 'ausente, fuera de la vista'.

M: $\mathrm{PM}{ }^{*} \boldsymbol{k} \boldsymbol{a} \boldsymbol{?}>\mathrm{Mk} \boldsymbol{k} \boldsymbol{a} \boldsymbol{?}$ 'ausente', Ni $\boldsymbol{k} \boldsymbol{a}$ 'conocido, no existente', Cho $\boldsymbol{k j} \boldsymbol{a} \sim \boldsymbol{k} \boldsymbol{i}$ 'alejándose, ido; muerto, consumido'.

Cf. también (89).

La gran mayoría de las semejanzas léxicas guaicurú-mataguayas ocurren no sólo entre lenguas o dialectos en contacto, sino en todas o en la mayoría de las lenguas de cada una de las dos familias, y resultan por tanto reconstruibles independientemente tanto para el PG como para el PM. Naturalmente, como resulta normal en situaciones de parentesco, se dan también casos en que -dentro de una de las dos familias- posibles cognados ocurren sólo en una lengua, como en los siguientes ejemplos:

(85) G: $\mathrm{PG}$ *inaqa $>\mathrm{Mb}<$ nicagaga> 'algarroba... blanca', < nicagag'igo > 'algarrobo... especie', Ab inieRa 'flor de algarrobo'.

M: Mk ininqa- $\boldsymbol{k}$ 'algarrobo blanco (Prosopis alba) ${ }^{35}$.

(86) G: PPT * poté- 'ser lento' > Pi ne-poté-ta-pege? 'lentamente', na-potá-\{an-aq 'tortuga', To pot-aj-k 'lento', n-pot-tak 'va lentamente', pota-Gan-aGat 'tortuga'.

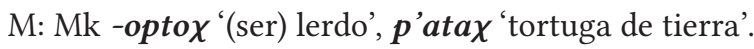

(87) G: PG *-n-ojén' > Ka -noe:n:, Mo -nojen -nohin, Pi -nojén, To -nojin 'llorar' M: Ni -ıjan 'llanto'.

(88) G: PGS *-kot- > Ab -kat- 'servir (a alguien)', Pi -kotfi-já 'ayudar', -kotf-aGan 'curar'.

M: Wi - $t$ f'ot- (dialecto weenhayek $-\boldsymbol{k}^{j}$ 'ot-) 'ayudar'.

(89) G: PG *t to > Ka dzo, Ab - ha, Mo so, Pi so?, To so 'distante, en movimiento'.

M: Wi tso 'lejano, visible'.

(90) G: Ka -itodi 'raíz'.

M: PM * $h^{w}$ etets > Mk fitets, Ni fetas, Cho $f^{w}$ etis, Wi $\boldsymbol{h}^{w}$ etes 'raíz'.

$\mathrm{G}: \mathrm{Mb}<-$ atiquelo > 'cerebro', <-atiquilo> 'meollo de la cabeza, sesos'.

M: PM *hetekiłV > Mk (vía metátesis) -exkitiła, Cho -tekiti 'cerebro, seso'.

$35 \mathrm{Mk}$ ininqak es un fitónimo sin cognados conocidos en las demás lenguas mataguayas. Pero el derivado PM “in $(\boldsymbol{a}) \boldsymbol{q} \boldsymbol{\Lambda} \boldsymbol{- p}$ 'verano', vease la serie (72), es etimológicamente *'época de algarrobo' (y, de hecho, el reflejo $\mathrm{Ni}$ ink $\boldsymbol{\Lambda} \boldsymbol{\text { Pp }}$ es glosado 'época de algarrobo' por Seelwische 1975:353), lo que indica que este nombre de planta debía estar presente ya en $\mathrm{PM}$, aunque solamente ha sobrevivido en una lengua actual. 
(92) G: Ab -oaoa 'morir'.

$\mathrm{M}: \mathrm{PM}{ }^{*}-\boldsymbol{w a h}^{w}>\mathrm{Mk}-\boldsymbol{w e f}$ 'estar cansado, cansarse', Ni -waf, Cho -wax 'muerto'.

(93) G: Pi awó 'mujer'.

M: PM " $\boldsymbol{a} \boldsymbol{h}^{w} \boldsymbol{u}>$ Mk $\boldsymbol{e} \boldsymbol{u} \boldsymbol{u}$, Cho (dialectos yowuwa y manjuy) $\boldsymbol{a} \boldsymbol{x}^{w} \boldsymbol{w} \boldsymbol{u} \boldsymbol{?} \sim \boldsymbol{a x u}$ ? 'mujer'.

Inclusive, en ocasiones, un eventual cognado se encuentra sólo en una lengua de cada una de las dos familias comparadas, como en:

(94) G: Ka -ote-di 'estrella'.

M: Mk foote-ki 'estrella'.

(95) G: Ka $\boldsymbol{e}: l: \boldsymbol{e}$ 'otro'.

M: Wi ef 'otro'.

Los eventuales cognados léxicos presentados se encuentran dentro de la parte del vocabulario que suele llamarse "no cultural" o "básica", y que se considera en general poco proclive a pasar en préstamo de una lengua a otra.

Es interesante, además, el hecho de que los eventuales cognados léxicos guaicurú-mataguayo mencionados incluyen una irregularidad compartida: la serie (18) constituye el imperativo supletivo del verbo de la serie (14). Irregularidades compartidas de este tipo son típicas en casos de parentesco remoto, y - por el contrario - no son frecuentes como préstamos.

Entre lenguas históricamente relacionadas, ocasionalmente un cognado existe en una de las familias emparentadas sólo como miembro de compuestos: un ejemplo de esto se daría en mataguayo en la serie (43).

\subsubsection{Semejanzas gramaticales}

Entre los posibles cognados gramaticales se encuentran los siguientes:

\section{Marcadores de persona}

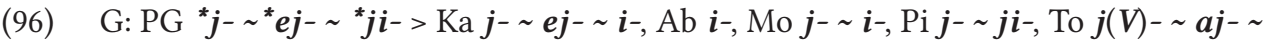
$\boldsymbol{i}-{ }^{\prime}{ }^{\mathrm{a}} \mathrm{p}$ p. sing. posesiva'.

M: PM *j(i)- > Mk ji- $\sim \boldsymbol{j}-\sim \boldsymbol{i}$-, Ni $\boldsymbol{i}-\sim \boldsymbol{j} \boldsymbol{i}$-, Cho $\boldsymbol{i}-\sim \boldsymbol{j}$-, Wi (dialecto weenhayek, sociolecto "familiar") $\boldsymbol{j}-\sim \boldsymbol{j} \boldsymbol{a}-\sim \mathbf{i} \boldsymbol{i}$ ' ' $1^{\mathrm{a}}$. p. sing. posesivo'.

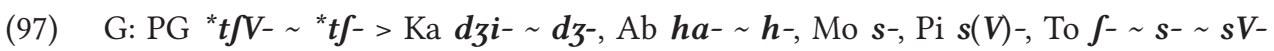
' 1 a p. sing. sujeto activo'.

M: PM ${ }^{\star} \boldsymbol{h} \boldsymbol{a}->$ Mk $\boldsymbol{h} \boldsymbol{V}-\sim \boldsymbol{h} \boldsymbol{a}^{-}$' $1^{\mathrm{a}}$ p. sing. sujeto', Ni $\boldsymbol{x} \boldsymbol{a}-\sim \boldsymbol{x}$ - ' $1^{\mathrm{a}}$ p. sing. sujeto activo', Cho $\boldsymbol{a}$ - $\boldsymbol{\varnothing}$ - ' $1{ }^{\text {a }}$ p. sing. sujeto activo', Wi (dialecto weenhayek, sociolecto "familiar") $\boldsymbol{h} \boldsymbol{a}-\sim \boldsymbol{a}$ - ' 1 a p. sing. sujeto'.

(98) G: PG ${ }^{\boldsymbol{*}} \boldsymbol{i}$ - ' 1 a p. sing. sujeto' ante los prefijos ${ }^{*} \boldsymbol{d}$ - 'diátesis estativa' y ${ }^{*} \boldsymbol{n}$ - 'diátesis media'.

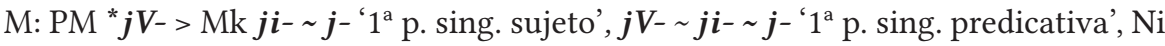




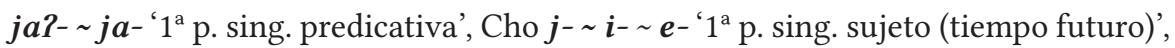
Wi $\boldsymbol{j a}^{-}$- ' $1^{\mathrm{a}}$ p. pl. sujeto', (dialecto weenhayek) $\mathbf{i j a} \boldsymbol{a}^{\text {' }} \mathbf{1}^{\mathrm{a}} \mathrm{p}$. pl. inclus. sujeto'.

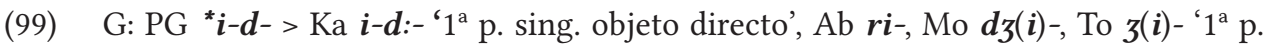
sing. sujeto inactivo'.

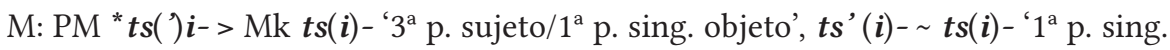
sujeto', Ni $t s\left(\right.$ ') $\boldsymbol{i}-\sim \boldsymbol{t} \boldsymbol{s}\left({ }^{\prime}\right)-$ ' $3^{\mathrm{a}}$ p. sujeto/1a. p. sing. objeto', $\boldsymbol{t s}(\boldsymbol{i})-$ ' $^{\mathrm{a}}$ p. sing. sujeto inactivo', Cho $s(i)-\sim t s(a)-$ ' 1 a $p$. sing. sujeto inactivo'.

(100) G: PG ${ }^{*}-\boldsymbol{i}>\mathrm{Ka}-\boldsymbol{i}, \mathrm{Ab}-\boldsymbol{i} \boldsymbol{i}, \mathrm{Mo}-\boldsymbol{i}$, To $-\boldsymbol{i}$ ' $1^{\mathrm{a}}$ p. sing. objeto indirecto'.

$\mathrm{M}: \mathrm{PM} 1{ }^{*}-\boldsymbol{j} \boldsymbol{i}>\mathrm{Mk}-\boldsymbol{j} \sim-\boldsymbol{j} \boldsymbol{i} \sim-\boldsymbol{j} \boldsymbol{V}, \mathrm{Ni}-\boldsymbol{j} \boldsymbol{i} \sim-\boldsymbol{j} \sim-\boldsymbol{j} \boldsymbol{a}$ ' $1^{\mathrm{a}} \mathrm{p}$. sing. objeto indirecto'.

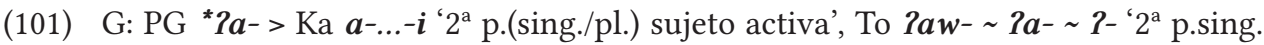
sujeto activa'.

M: PM ${ }^{*} \boldsymbol{a}^{-} \sim{ }^{*} \boldsymbol{\emptyset}->\mathrm{Mk} \boldsymbol{V}-\sim \boldsymbol{a}-\sim \boldsymbol{\emptyset}^{-}$' $2^{\mathrm{a}}$ p. sing. sujeto (modo no indicativo)', Ni $\boldsymbol{a}-\sim \varnothing$ - ' $2^{\mathrm{a}}$ p. sing. sujeto (modo subjuntivo)', Cho $\boldsymbol{a}-\sim \varnothing^{-}$' $2^{\mathrm{a}}$ p. sing. sujeto (tras negación)'.

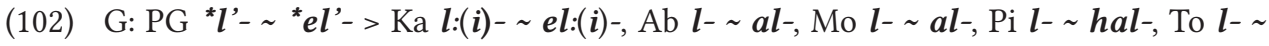
$\boldsymbol{a l}-{ }^{\prime} 3^{\mathrm{a}} \mathrm{p}$. posesiva'.

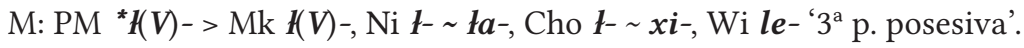

(103) G: PG ${ }^{\boldsymbol{*}} \boldsymbol{j}(\boldsymbol{i})->\mathrm{Ka} \boldsymbol{j}-\sim \boldsymbol{i}$-, Ab $\boldsymbol{i}$-, Mo $\boldsymbol{j}-\sim \boldsymbol{i}$-, Pi $\boldsymbol{j}(\boldsymbol{i})-$, To $\boldsymbol{j}-\sim \boldsymbol{i}-{ }^{-} 3^{\text {a }}$ p. sujeto activo'.

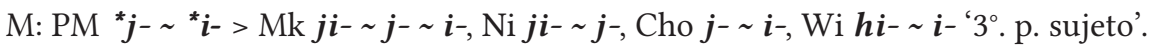

(104) G: PG * Ø- > Ka Ø-, Ab Ø-, Mo Ø-, Pi Ø-, To Ø- '3ª p. sujeto activo’.

M: PM * Ø-> Mk Ø-, Ni Ø-, Cho Ø-, Wi Ø- '3 3 p. sujeto activo'.

(105) G: $\mathrm{PG}{ }^{*}-\boldsymbol{e}>\mathrm{Ka}-\boldsymbol{e}, \mathrm{Ab}-\boldsymbol{e}, \mathrm{Mo}-\boldsymbol{e}, \mathrm{Pi}-\dot{e}$, To $-\boldsymbol{e}$ ' $3^{\mathrm{a}}$ p. objeto indirecto'.

$\mathrm{M}: \mathrm{PM}{ }^{*}-\boldsymbol{e}>\mathrm{Mk}-\boldsymbol{i} \sim-\boldsymbol{V}, \mathrm{Ni}-\boldsymbol{e} \sim-\boldsymbol{a}, \mathrm{Wi}-\boldsymbol{P} \boldsymbol{e}^{\text {' }} 3^{\mathrm{a}} \mathrm{p}$. objeto indirecto'.

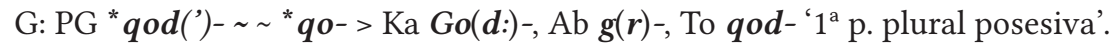

M: PM *kats(')- > Ni kas- kats(i)- kats'(i)- '1 $1^{\text {a }}$ p. pl. inclusivo posesiva', Cho (dialecto manjuy) $k a t s(V)-\sim \operatorname{kas}(V)-{ }^{\prime a} 1^{a}$ p. pl. posesiva'.

G: PG * $\boldsymbol{q} \boldsymbol{o}(-\boldsymbol{d})->\mathrm{Ka} G \boldsymbol{o}(\boldsymbol{d}:)-$, Ab $\boldsymbol{g} \boldsymbol{r}-\ldots-\boldsymbol{a q}$, To $\boldsymbol{q} \boldsymbol{o}-$ ' $^{\mathrm{a}}$ p. pl. inactiva'36.

M: PM *kats- > Ni kas- ' $1{ }^{a}$ p. pl. inclusivo sujeto predicativo', Cho kasts $(a)$ kats- kas- ' 1 a p. pl. sujeto predicativo'.

(108) G: PG ${ }^{*} \boldsymbol{n}-\sim{ }^{*} \boldsymbol{e n}->$ Ka $\boldsymbol{n}(\boldsymbol{i})-\sim \boldsymbol{e n}(\boldsymbol{i})-$, Ab $\boldsymbol{n}$-, Mo $\boldsymbol{n}$-, Pi $\boldsymbol{n}$-, To $\boldsymbol{n}$ - $\sim \boldsymbol{a n}$ - 'posesivo impersonal ${ }^{37}$.

36 Los prefijos de primera persona plural de las lenguas qom que presentan vocal $\boldsymbol{a}$ (To $\boldsymbol{q a d}$-, etc), antes considerados alomorfos de $\mathbf{q o}$ - etc., han sido analizados recientemente como compuestas de $\boldsymbol{q}(\boldsymbol{o})$ - ' $1^{\mathrm{a}}$. p. pl.' + $\boldsymbol{a} \boldsymbol{d}$ - (y variantes) '2a . p. sing.' (Carpio 2012:105-108).

37 En guaicurú este prefijo también funciona como alienabilizador. Messineo (2002:70) explica la identidad originaria con el marcador de diátesis media * $\boldsymbol{n}$ - de la serie (125): 'El participante medio instiga, ejecuta y controla una acción que íntimamente lo involucra... De la misma manera, el poseedor de una construcción con el marcador de alienabilidad / $\boldsymbol{n}$-/ presupone un participante agente que debió realizar la acción de adquirir al poseído 
M: PM * $\boldsymbol{n}^{->}$Mk $\boldsymbol{n}$ - (prefijo poco frecuente), Ni $\boldsymbol{n}-\sim \boldsymbol{n} \boldsymbol{a}^{-}$, Cho $\boldsymbol{n}-\sim \boldsymbol{n i} \boldsymbol{i}^{-} \boldsymbol{n} \boldsymbol{a}^{-} \sim \boldsymbol{i n} \boldsymbol{-}^{-}$ $\boldsymbol{i m}$ - i- 'posesivo impersonal'.

Cf. también (128) y (135).

\section{Sufijos de número}

(109) G: $\mathrm{PG}^{*}-\boldsymbol{V} \boldsymbol{d} \boldsymbol{i}>\mathrm{Ka}-(\boldsymbol{a}) \boldsymbol{d} \boldsymbol{i}, \mathrm{Ab}-\boldsymbol{r i}$, Mo -ri, Pi $-\boldsymbol{d i}$, To -(V) $\boldsymbol{z} \boldsymbol{i}$ 'plural nominal'.

$\mathrm{M}: \mathrm{PM} *-(V) t \boldsymbol{s}>\mathrm{Mk}-V t \boldsymbol{s} \sim-\boldsymbol{t s}, \mathrm{Ni}-V \boldsymbol{s} \sim-\boldsymbol{s}$, Cho $-V \boldsymbol{s} \sim-\boldsymbol{s}$, Wi $-\boldsymbol{e s} \sim-\boldsymbol{s}$ '(alomorfo de) plural nominal'.

(110) G: PG ${ }^{*}-\boldsymbol{a l}$ '(i) > Ka $-(\boldsymbol{a}) \boldsymbol{l}: \boldsymbol{i}$, Ab - $\boldsymbol{l}$ 'plural nominal', Mo - $\boldsymbol{l}$, Pi $-\boldsymbol{l}$ 'paucal', To $-l$ 'distributivo'.

$\mathrm{M}: \mathrm{PM}^{*}-(\boldsymbol{V}) \boldsymbol{l}>\mathrm{Mk}-\boldsymbol{l}$, Ni $-\boldsymbol{k}\left(<^{*}-\boldsymbol{k} \boldsymbol{l}\right)$, Cho $-(\boldsymbol{V}) \boldsymbol{l}$, Wi $-\boldsymbol{l}$ '(alomorfo de) sufijo de plural nominal'.

(111) G: PG * ${ }^{*} \boldsymbol{w a}>\mathrm{GN}$ : Ka $-\boldsymbol{w a}$, Ab -oo -o, Mo $-\boldsymbol{w a}$ 'plural de demostrativos', To - $\boldsymbol{w a}$ 'dual de demostrativos'.

M: PM *-wa > Mk -we? '(alomorfo de) plural de demostrativos', Ni - wa 'plural nohumano de demostrativos', Cho - wa 'plural no-humano de demostrativos'.

(112) G: PGS ${ }^{*}-\boldsymbol{i}>\mathrm{Ab}-\boldsymbol{i} \sim-\boldsymbol{e}, \mathrm{Mo}-\boldsymbol{i}, \mathrm{Pi}-\boldsymbol{i}$, To $-\boldsymbol{i}$ 'plural de segunda persona sujeto'.

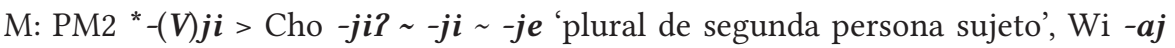
'plural de segunda persona sujeto/objeto'.

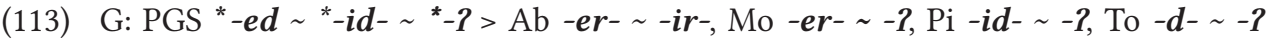
'plural de tercera persona sujeto'.

M: PM2 * ${ }^{*} \boldsymbol{e s} \sim{ }^{*}-\boldsymbol{i s}>$ Cho $-\boldsymbol{i s}(\boldsymbol{i}) \sim-\boldsymbol{j i s} \sim-\boldsymbol{s i} \sim-\boldsymbol{e s}$ 'plural de $3^{\mathrm{a}}$ p. sujeto', Wi $-\boldsymbol{e s}$ 'plural (en verbos predicativos)'.

Cf. también (129).

\section{Sufijos aplicativos y direccionales}

(114) G: PG ${ }^{*}-\boldsymbol{m}>\mathrm{Ka}-\boldsymbol{m a}$ '(alomorfo de) dativo', MoS -om, MoN - $\boldsymbol{e} \boldsymbol{m} \sim \boldsymbol{-} \boldsymbol{m}, \mathrm{Pi}-\boldsymbol{m}$, To $-\boldsymbol{e} \boldsymbol{m} \sim-\boldsymbol{m}$ ' (alomorfo de) benefactivo'.

M: PM ${ }^{*}-\boldsymbol{m}>\mathrm{Mk}-\boldsymbol{m}$ sufijo que 'indica beneficio o perjuicio, resultado o relación (en cuanto)', $\mathrm{Ni}-\boldsymbol{m}$ sufijo de 'objeto indirecto', Wi $-\boldsymbol{a}-\boldsymbol{m}$ ' $2^{\mathrm{a}}$ p. objeto'.

(115) G: PG *-g'i > Ka - $\boldsymbol{n i - g}$ : 'locativo', Mb < tigi $>$ 'en', Mo -(i) $\boldsymbol{g i}$ 'en, dentro', Pi - $\boldsymbol{j i}$ 'hacia abajo (y hacia adentro)', To -(i)gi 'en, dentro'.

M: PM * $\boldsymbol{h} \boldsymbol{h} \boldsymbol{j}>\mathrm{Mk}-\boldsymbol{x} \boldsymbol{i \boldsymbol { i }}$ 'en, en el interior de', Ni $-\boldsymbol{f} \boldsymbol{i}$ 'en', Cho - $\boldsymbol{h i}(?)$ 'locativo', Wi -hi 'en, dentro'.

(116) G: PG * ${ }^{*} \boldsymbol{e} \boldsymbol{k}^{\prime} \boldsymbol{e}>\mathrm{Ka}-(\boldsymbol{e}) \boldsymbol{k} \boldsymbol{e}, \mathrm{Ab}-\boldsymbol{o o k}$, Mo -(w)ek, To - wek 'hacia afuera'.

M: PM * $(-) \boldsymbol{h}^{w} \boldsymbol{e} \boldsymbol{k}^{\prime} \boldsymbol{e} \sim{ }^{*}-\boldsymbol{k}^{\prime} \boldsymbol{e}>\mathrm{Mk}-\boldsymbol{f} \boldsymbol{i} \boldsymbol{k}$ 'i, Ni fetf'e 'afuera', Wi -t $\boldsymbol{e} \boldsymbol{e}$ 'externo, hacia afuera'.

para su propio beneficio'. 


\section{Sufijos de derivación nominal}

(117) G: $\mathrm{PG}{ }^{*}-\boldsymbol{i} \boldsymbol{k} \boldsymbol{o}>\mathrm{Ka}-\boldsymbol{i g o}, \mathrm{Ab}-\boldsymbol{i} \boldsymbol{k}$, Mo $-\boldsymbol{i} \boldsymbol{k}$, $\mathrm{Pi}-\boldsymbol{i} \boldsymbol{k}$, To $-\boldsymbol{i} \boldsymbol{k}$ 'clasificador nominal (plantas leñosas)'.

$\mathrm{M}: \mathrm{PM}{ }^{*}-\boldsymbol{u} \boldsymbol{k}>\mathrm{Mk}-(\boldsymbol{u}) \boldsymbol{k}$, Ni $-\boldsymbol{j} \boldsymbol{u} \boldsymbol{k} \sim-\boldsymbol{u} \boldsymbol{k}$, Cho $-\boldsymbol{u} \boldsymbol{k}$, Wi $-(\boldsymbol{u}) \boldsymbol{k} \sim-\boldsymbol{k}^{\boldsymbol{w}}$ 'clasificador nominal (plantas)'.

(118) G: PG *-naqa > Ka -naGa 'nominalizador', Pi -naq, To - $\boldsymbol{n} \boldsymbol{a q}$ 'nominalizador ("que tiene mucho...”')

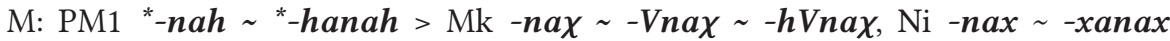
'nominalizador frecuentativo'.

(119) G: PG *t ${ }^{*}$ 'aqa > Ka -t $\int \boldsymbol{a} G \boldsymbol{a}, \mathrm{Ab}-\boldsymbol{h a q} \sim-\boldsymbol{h a R}$-, Mo -saq, To -saq 'nominalizador ("que hace o tiene mucho...")'.

$\mathrm{M}: \mathrm{PM}{ }^{*}-\boldsymbol{t s} \boldsymbol{a h} \sim{ }^{*}-\boldsymbol{a h}>\mathrm{Mk}-\boldsymbol{t s a \chi} \sim-\boldsymbol{a \chi}$, Wi $-\boldsymbol{s} \boldsymbol{a h} \sim-\boldsymbol{a h}$ 'nominalizador'.

(120) G: PG *-ek > Mb <-igi> "participio", Ab - $\boldsymbol{e}$ 'relativo a', To - $\boldsymbol{e}$ k 'nominalizador (resultativo y objetivo)'.

M: PM1 *hate > Mk -het, $\mathrm{Ni}-\boldsymbol{x a t} \sim$-xate 'nominalizador (instrumental)'.

(121) G: PG * $-\boldsymbol{e} \boldsymbol{k}>\mathrm{Mb}<-\boldsymbol{i g i}>$ "participio", Ab - $\boldsymbol{e} \boldsymbol{k}$ 'relativo a', To - $\boldsymbol{e}$ ' nominalizador deverbal (resultativo y objetivo)'.

M: $\mathrm{PM}{ }^{*}-\boldsymbol{e} \boldsymbol{k} \sim{ }^{*}-\boldsymbol{i} \boldsymbol{k}>\mathrm{Mk}-\boldsymbol{e} \boldsymbol{k} \sim-\boldsymbol{i} \boldsymbol{k}$ 'participio', $\mathrm{Ni}-\boldsymbol{a t} \int \sim-\boldsymbol{e t} \int \sim-\boldsymbol{i t} \int$ 'nominalizador de “sujeto pasivo”', Wi $-\boldsymbol{a} \boldsymbol{k} \sim \boldsymbol{- \boldsymbol { k }} \sim \boldsymbol{- i \boldsymbol { k }}$ 'nominalizador (nombres de acción, de agente)', (dialecto weenhayek) - ek 'participio'.

\section{Sufijos de género}

(122) G: $\mathrm{PG}{ }^{*}-\dot{e}>\mathrm{Ka}-\boldsymbol{e}, \mathrm{Ab}-\boldsymbol{e}, \mathrm{Mo}-\boldsymbol{e}, \mathrm{Pi}-\boldsymbol{e}$, To -e '(alomorfo de) femenino'.

$\mathrm{M}: \mathrm{PM}^{*}-\boldsymbol{e}>\mathrm{Mk}-\boldsymbol{i} \boldsymbol{2} \sim-\boldsymbol{e}$, $\mathrm{Ni}-\boldsymbol{e}$, Cho -e?, Wi -e '(alomorfo de) femenino'.

(123) G: $\mathrm{PG}^{*}-\boldsymbol{o} \sim{ }^{*}$-ó > Ka -o, Mo -o, Pi -o -ó, To -o '(alomorfo de) femenino'.

$\mathrm{M}: \mathrm{PM}^{*}{ }^{\boldsymbol{\Lambda}} \boldsymbol{\Lambda}>\mathrm{Mk}-\boldsymbol{a}$, $\mathrm{Ni}-\boldsymbol{\Lambda}, \mathrm{Wi}-\boldsymbol{\Lambda}$ '(alomorfo de) femenino'.

(124) G: $\mathrm{PG}{ }^{*}-\boldsymbol{i} \boldsymbol{k}>\mathrm{Ka}-\boldsymbol{i g} \boldsymbol{i}, \mathrm{Ab}-(\boldsymbol{i}) \boldsymbol{k}$, Mo $-(\boldsymbol{i}) \boldsymbol{k}$, $\mathrm{Pi}-(\boldsymbol{i}) \boldsymbol{k}$, To $-(\boldsymbol{i}) \boldsymbol{k}$ 'masculino'.

$\mathrm{M}: \mathrm{PM}^{*}-(\boldsymbol{V}) \boldsymbol{k}(\boldsymbol{i})>\mathrm{Ni}-\mathbf{k}$, Cho $-\boldsymbol{k} \sim-\boldsymbol{k i}$, Wi $-(V) \boldsymbol{k} \sim-(V) \boldsymbol{k}^{w}$ 'masculino'.

\section{Otros elementos gramaticales}

(125) G: PG * $\boldsymbol{n}$-> Ka $\boldsymbol{n}$ - ni-, Ab $\boldsymbol{n}$-, Mo $\boldsymbol{n}$-, Pi $\boldsymbol{n}$-, To $\boldsymbol{n}$ - 'diátesis media'.

M: PM * $\boldsymbol{n}$-> Mk $\boldsymbol{n}$ - 'dirección hacia el locutor' (prefijo fosilizado en algunos verbos de movimiento), Ni $\boldsymbol{n}$ - $\sim \boldsymbol{n} \boldsymbol{a}$ - 'dirección hacia el locutor', 'acción refleja', Cho $\boldsymbol{n i}$ 'reflexivo', Wi $\boldsymbol{n}$ - 'diátesis media'.

(126) G: $\mathrm{PG}{ }^{*}-\boldsymbol{a q}-\boldsymbol{a t} V \sim{ }^{*}-\boldsymbol{a t V}>\mathrm{Ka}-\mathrm{Gad} \sim-\boldsymbol{a d}$, Ab -Rat $\sim-\boldsymbol{a t}$, Mo -aGat $\sim-$ Gat $\sim-$ qat $~$ -at, $\mathrm{Pi}-\boldsymbol{a}$ Sat $\sim-\boldsymbol{S a t} \sim-\boldsymbol{a t}$, To -aGat $\sim-G a t \sim-a t$ 'transitivizador instrumental'. 


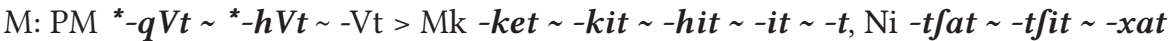

$\sim-\boldsymbol{a t}$, Cho -et, Wi -kat -hat (dialecto weenhayek -qat $\sim$-hat) 'causativo'.

G: $\mathrm{PG}{ }^{*}$-én > Ka -en; Ab -en, Mo -en, Pi -én, To -en 'verbalizador'.

$\mathrm{M}: \mathrm{PM}{ }^{*}-\boldsymbol{n}>\mathrm{Mk}-\boldsymbol{n}, \mathrm{Ni}-\boldsymbol{n} \sim-\boldsymbol{i n} \sim-\boldsymbol{a n}, \mathrm{Wi}-\boldsymbol{n}$ 'verbalizador'.

Cf. también (130).

Tal como ocurre con las semejanzas léxicas, entre los ítems gramaticales hay casos en que la posible cognación ocurre (o hasta ahora ha sido documentada) sólo en una lengua de una de las dos familias:

(128) G: $\mathrm{PG}{ }^{*}-\boldsymbol{q o}>\mathrm{Ka}-\mathrm{Go}$, Mo -oGo, To -oGo ' $1^{\text {a }}$ p. pl. objeto indirecto'.

$\mathrm{M}: \mathrm{Ni}-x \boldsymbol{o}$ ?' $^{\mathrm{a}} \mathrm{a}$ p. pl. inclus. objeto indirecto'.

(129) $\mathrm{G}: \mathrm{PG}{ }^{*}-\boldsymbol{a q} \boldsymbol{a}>\mathrm{Ka}-\boldsymbol{a} G \boldsymbol{a} \sim-G \boldsymbol{a}$ 'plural de primera y tercera personas sujeto', $\mathrm{Ab}-a q \sim-q a \sim-a R-$, Mo $-a q \sim-a G a$, $\mathrm{Pi}-\boldsymbol{S a} \sim-a q o \sim-a q \sim-q a$, To $-\boldsymbol{q} \sim-a G a \sim$ $-\boldsymbol{a q}$ 'plural de primera persona sujeto'.

M: Cho $-(\boldsymbol{V}) \boldsymbol{k} \sim \sim-(\boldsymbol{x}) \boldsymbol{a}$ 'plural de primera persona sujeto'.

(130) G: Ab $\boldsymbol{m}$ - 'encabezador de interrogación total'.

M: $\mathrm{PM}{ }^{*} \boldsymbol{m} \boldsymbol{e}>\mathrm{Mk} \boldsymbol{m e}$, Cho $\boldsymbol{m a} \sim \boldsymbol{m i} \sim \boldsymbol{m}$ 'encabezador de interrogación total' ${ }^{38}$.

El isomorfismo gramatical guaicurú-mataguayo implica paralelismos idiosincráticos tan llamativos como la identidad o cuasi-identidad formal entre las marcas de $1^{\mathrm{a}}$ p. sing. posesiva y sujeto - series (96) y (98) - y de $3^{\mathrm{a}} \mathrm{p}$. sujeto - serie (103).

En ocasiones, los detalles de la alomorfía de algunos de los posibles cognados gramaticales muestran semejanzas mucho más notables de lo que a primera vista parecería. En la serie (104), por ejemplo, es interesante que los alomorfos $\varnothing$-de $3^{\text {a }}$. p. sujeto ocurran en algunos contextos muy similares en guaicurú y en mataguayo, por ejemplo ante los prefijos PG *n- 'voz media' y su eventual cognado - cf. la serie (125) - PM *n- 'voz media' / 'direccional cislocativo', como en:

G: Ka Ø-ni-qoGeti 'se detiene', To Ø-n-amaq 'lo manda hacia acá', etc.

M: Ni Ø-n-axaft 'trae de vuelta', Wi Ø-n-am 'llega', etc.

Una semejanza en alomorfos $\varnothing$-de $3^{\text {a }}$ persona podría ser casual (alomorfos cero de marcadores de tercera persona son frecuentes en las lenguas del mundo); pero la coincidencia en los usos específicos de tales alomorfos es digna de consideración.

En la serie (108), el PG presenta dos alomorfos del prefijo de poseedor indefinido (siendo la segunda variante la menos frecuente, sobre todo en GS donde ocurre sólo ante algunas raíces monosilábicas que empiezan con vocal), frente a una única forma reconstruida para el PM:

PG *n- *en-, PM *n-.

38 En Mk también encabezador de interrogación indirecta (con verbos de conocimiento o percepción). 
Pero en mataguayo existe al menos una serie cognada:

PM *hin-aws-p 'época de flores' > Mk xinawap, Ni fns wap, Cho nawop y Wi (i) nawop 'primavera',

que parece suponer un alomorfo ${ }^{*} \boldsymbol{h i n}$-del prefijo de poseedor indefinido. Esto implicaría que en PM habrían existido dos alomorfos ( ${ }^{*} \boldsymbol{n}$ - y ${ }^{*} \boldsymbol{h} \boldsymbol{i n}$-) del prefijo de poseedor indefinido, paralelizando lo que ocurría en PG:

(108b) PG ${ }^{*} \boldsymbol{n}-\sim{ }^{*} \boldsymbol{e n}-, \mathrm{PM}{ }^{*} \boldsymbol{n}-\sim{ }^{*} \boldsymbol{h i n}$ -

Y PM *hin-fue evidentemente la variante menos frecuente, tal como sucede con el alomorfo *en- en PG.

Para el sufijo nominalizador de la serie (119), en PG hay una única forma frente a dos alomorfos en la forma correspondiente del PM:

$\mathrm{PG}^{*}-t \boldsymbol{f}^{\prime} \boldsymbol{a q a}, \mathrm{PM}{ }^{*}-t s a h \sim^{*}-\boldsymbol{a h}$.

Sin embargo, en base a evidencia interna guaicurú, he sugerido en

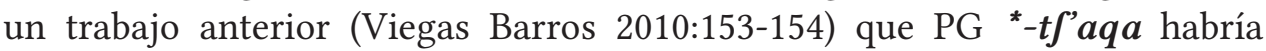
alternado originalmente con un alomorfo ${ }^{*}-a q a$, con lo que la semejanza entre las protoformas de ambas familias en esta serie se vuelve más notable:

(119b) $\quad \mathrm{PG}{ }^{*}-t \int^{3} a q a \sim^{*}-a q a, \mathrm{PM}{ }^{*}-t s a h \sim^{*}-a h$.

En general, las semejanzas gramaticales guaicurú-mataguayo, son de un tipo muy diferente a las que están documentadas en situaciones de Sprachbund típicas. La relación entre las familias guaicurú y mataguaya parece la imagen inversa, por ejemplo, de lo que ocurre entre las familias quechua y aimara, en las que la inmensa mayoría de las semejanzas gramaticales implican elementos fonológicamente muy distintos (véase, entre otros, Calvo Pérez 1994).

\subsubsection{Posibles gramaticalizaciones en una o otra de las familias (o en ambas independientemente)}

Algunas delas series eventualmente cognadas implicarían gramaticalización en todas o en algunas de las lenguas de una de las dos familias, como sucede en las siguientes series:

(131) G: PG *-wot > Mb <ibotigi> 'adentro', Ab -aoate 'morada', Mo -wot- -wo?'casa, familia', To -wo?' casa'.

$\mathrm{M}: \mathrm{PM}{ }^{*}$-wet $>\mathrm{Mk}-\boldsymbol{w e t}, \mathrm{Ni}-\boldsymbol{w a t} \sim-\boldsymbol{x a}$-wat, , Cho -wet, Wi -wet 'lugar donde hay...' (sufijo locativo) ${ }^{39}$.

G: PG *-Pawéke *-Pawék'e > Ka -t-aweke, Ab -aoge -aok -t-aoge, Mo -aloge

$\sim$-wa?ke, Pi -wák, To -wak 'cuantificador total' (sufijo de demostrativos).

M: PM *wek > Mk week, Cho wikjej 'todos', Wi wek 'juntos'.

39 En M2 también se encuentran los sustantivos Cho wet wit 'casa', Wi wet 'lugar'. 
(133) G: PQ *-?ot > Mo -?ot, Pi -?ot, To - 1 ot 'hacia abajo' (sufijo direccional).

M: PM ${ }^{*}-(\boldsymbol{n})-\boldsymbol{\Lambda} \boldsymbol{t}>\mathrm{Ni}-\boldsymbol{\Lambda} \mathbf{t} \boldsymbol{t}-\boldsymbol{x a t}$ 'dejar caer', $-\boldsymbol{n}-\boldsymbol{\Lambda} \boldsymbol{t}$ 'dejarse caer', Wi (dialecto weenhayek) -n-at 'caer'.

En ciertos casos, la gramaticalización habría ocurrido independientemente en las lenguas mataguayas y en algunas lenguas de la familia guaicurú:

(134) G: PG *-atf'ap > Ka -at fabi 'orilla, al lado', MoS (Paucke) <-zop> 'alrededor', $\mathrm{Pi}$-sop 'en círculos', To -sop 'debajo de (alrededor)' ${ }^{40}$.

$\mathrm{M}: \mathrm{PM}{ }^{*}-\boldsymbol{h} \boldsymbol{V} \boldsymbol{p}>\mathrm{Mk}-\boldsymbol{x u p}, \mathrm{Ni}-\boldsymbol{x o p}$, Cho -hap 'alrededor'.

(135) G: PG * $\boldsymbol{q}$ 'o > Ka oqo 'gente', 'persona', Mo $\boldsymbol{q} \boldsymbol{o}$-, Pi $\boldsymbol{q} \boldsymbol{o}$ - 'agente desconocido', To qo- 'agente desconocido, diátesis pasiva'.

M: PM * $\boldsymbol{q}$ ' $\boldsymbol{a}$ - > Mk $\boldsymbol{q}$ ' $\boldsymbol{e}$-, Ni $\boldsymbol{k}$ ' $\boldsymbol{a}$-, Wi $\boldsymbol{q} \boldsymbol{a}$ - 'alienabilizador'.

Cf. además las notas 14 y 28.

Parece difícil interpretar semejanzas de este tipo como préstamos.

\subsubsection{Correspondencias fonológicas}

A partir de las precedentes series eventualmente cognadas, es posible establecer tentativamente las siguientes pautas de correspondencias fonológicas:

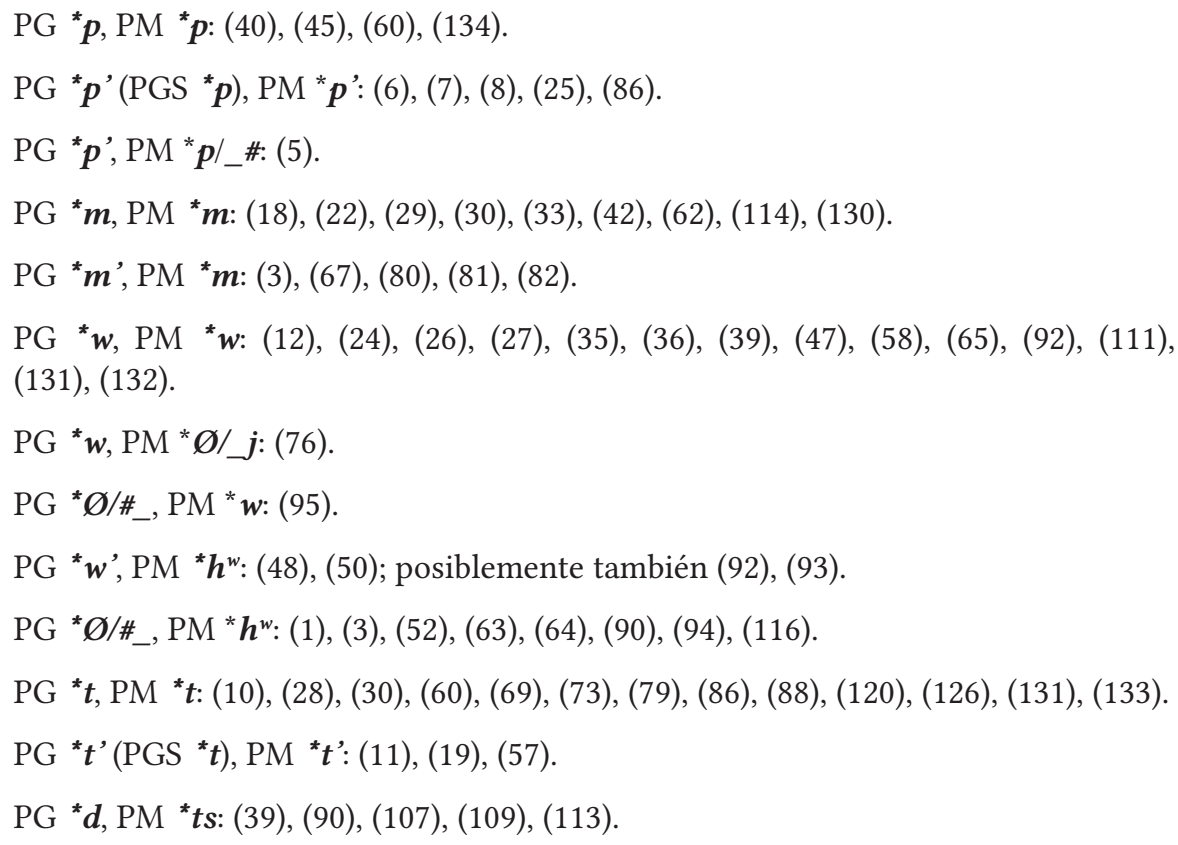

40 En toba antiguo existían formas todavía no gramaticalizadas de este ítem, llamadas "preposiciones" por Bárcena: <asopotetahé> 'junto a otra cosa', <asopotetát > $<$ assopotetát $>$ 'cerca, o junto a otra cosa'. 
$\mathrm{PG}{ }^{*} \boldsymbol{d} \sim{ }^{*} \boldsymbol{d}^{\prime}, \mathrm{PM}{ }^{*} \boldsymbol{t} \boldsymbol{s} \sim{ }^{*} \boldsymbol{t} \boldsymbol{s}$ : (99), (106).

PG ${ }^{*}$ ts, PM *ts: (32), (63).

$\mathrm{PG}^{*} t \boldsymbol{s}^{\prime}, \mathrm{PM}{ }^{*} s:(44)$.

PG ${ }^{*} \boldsymbol{n}, \mathrm{PM}{ }^{*} \boldsymbol{n}$ : (4), (9), (27), (31), (68), (72), (85), (108), (125), (118), (127).

PG * $\boldsymbol{n}^{\prime}, \mathrm{PM}{ }^{*} \boldsymbol{n}$ : (21), (53), (74), (87).

PG ${ }^{*} \boldsymbol{l}, \mathrm{PM}{ }^{*} \boldsymbol{l}$ : (25), (26), (28), (33), (47), (56), (66), (74), (78).

PG ${ }^{*} \boldsymbol{l}^{\prime}$ (PGS $\left.{ }^{*} \boldsymbol{l}\right), \mathrm{PM}^{*} \boldsymbol{t}$. (49), (73), (75), (82), (95), (102); posiblemente también (2), (35), (65), (91).

PG ${ }^{*} \boldsymbol{l}$, PM ${ }^{*} \boldsymbol{l} / \_$: (110).

$\mathrm{PG}^{*} t \boldsymbol{t}, \mathrm{PM}{ }^{*} t s:(89)$.

$\mathrm{PG}^{*} \boldsymbol{t} \boldsymbol{\int}\left(\mathrm{PGS}{ }^{*} \boldsymbol{s}\right), \mathrm{PM}{ }^{*} \boldsymbol{h}:(70),(97),(134)$.

$\mathrm{PG}^{*} t \int^{\prime}, \mathrm{PM}{ }^{*} t \boldsymbol{s}^{\prime}:(61)$.

$\mathrm{PG}^{*} t \int^{\prime}\left(\mathrm{PGS}{ }^{*} \boldsymbol{s}\right), \mathrm{PM}{ }^{*} \boldsymbol{s}:(9),(17),(77)$.

PG ${ }^{*} j$, PM ${ }^{*} j$ : (1), (34), (44), (52), (62), (64), (66), (76), (80), (87), (96), (103).

$\mathrm{PG}{ }^{*} \varnothing, \mathrm{PM} * \boldsymbol{j} / \boldsymbol{i}:(16),(99),(100),(112),(115)$.

$\mathrm{PG}{ }^{*} \varnothing / C_{-}, \mathrm{PM}{ }^{*} j:(63)$.

$\mathrm{PG}{ }^{*} \boldsymbol{j}, \mathrm{PM}{ }^{*} \varnothing / \boldsymbol{\gamma}:(45)$.

$\mathrm{PG}{ }^{*} \boldsymbol{j}, \mathrm{PM}{ }^{*} \boldsymbol{j}:(20)$.

PG ${ }^{*} \boldsymbol{k}$ (PGS * $\boldsymbol{k} / \#$ _, ${ }^{*} \boldsymbol{g}$ ), PM * $\boldsymbol{k}$ : (13), (14), (26), (61), (70), (79), (91), (117), (121), (124), (132).

PG ${ }^{*} \boldsymbol{k}, \mathrm{PM}{ }^{*} \boldsymbol{h}$ : (52), (55), (68).

PG ${ }^{*} \boldsymbol{k}$ '(PGS $\left.{ }^{*} \boldsymbol{k}\right)$, PM ${ }^{*} \boldsymbol{k}$ : (17), (51), (88), (116).

PG ${ }^{*} \boldsymbol{g}, \mathrm{PM}^{*} \boldsymbol{h}:(6),(71),(115)$.

PG * $\boldsymbol{q}, \mathrm{PM}{ }^{*} \boldsymbol{q}$ : (40), (44), (53), (69), (72), (74), (85), (106), (107), (126), (129).

PG * $\boldsymbol{q}, \mathrm{PM}{ }^{*} \boldsymbol{h}$ : (17), (37), (49), (61), (74), (118), (119), (120), (128), (129).

$\mathrm{PG}{ }^{*} \boldsymbol{q}^{\prime}, \mathrm{PM}{ }^{*} \boldsymbol{q}^{\prime}:$ (9), (36), (135).

$\mathrm{PG}^{*}$ ?, PM *?: (12), (24).

PG *2, PM * Ø: (13), (23), (26), (29), (30), (31), (32), (42), (55), (57), (75), (76), (81), (101), (132), (133).

PG * Ø, PM * $7 / \#$ \#: (1), (19), (21), (22), (34), (50), (59), (60), (74), (83), (84).

PG * $\varnothing,{ }^{*}$ ?, PM *h: (15), (16), (30), (36), (37), (38), (46), (53), (54), (56), (86), (91), (108b), (126).

PG * $\boldsymbol{a}, \mathrm{PM}$ * $\boldsymbol{a}$ : (2), (4), (5), (7), (8), (10), (11), (16), (19), (22), (30), (31), (37), (40), (44), (45), (48), (53), (59), (60), (64), (69), (70), (72), (74), (75), (76), (81), (82), (83), (84), (85), 
(92), (93), (101), (111), (119), (129), (135).

$\mathrm{PG} * \boldsymbol{a}, \mathrm{PM}{ }^{*} \boldsymbol{\Lambda} / \boldsymbol{C}_{[+\mathrm{GRAVE}]}$ : (3), (9), (35), (36), (49), (55), (58), (72).

$\mathrm{PG} * \boldsymbol{a}, \mathrm{PM}{ }^{*} \boldsymbol{o} / \boldsymbol{C}_{[+\mathrm{GRAVE}]}:(17),(25)$.

$\mathrm{PG} * \boldsymbol{o} / C_{[+\mathrm{GRAVE}]}, \mathrm{PM} * \boldsymbol{a}:(41)$.

$\mathrm{PG} * \boldsymbol{o} \sim{ }^{*} \boldsymbol{a} / \boldsymbol{C}_{[+\mathrm{GRAVE}]}, \mathrm{PM}{ }^{*} \boldsymbol{a}:(106),(107),(135)$.

PG ${ }^{*} \boldsymbol{a}, \mathrm{PM}{ }^{*} \boldsymbol{e}:$ (1), (26), (39), (70).

PG ${ }^{*} a / \#^{41}$, PM Ø: (5), (48), (118), (119).

$\mathrm{PG}^{*} \boldsymbol{e}, \mathrm{PM}{ }^{*} \boldsymbol{e}$ : (13), (15), (17), (26), (27), (33), (47), (51), (56), (61), (66), (71), (73), (78), (94), (95), (105), (116), (121), (122), (132).

PG *e, PM *i: (28), (43), (49), (76), (108b).

PG ${ }^{*} \boldsymbol{e} \sim{ }^{*} i, \mathrm{PM}{ }^{*} \boldsymbol{e} \sim{ }^{*} \boldsymbol{i}:(14),(113)$.

$\mathrm{PG}^{*} \boldsymbol{e}, \mathrm{PM}{ }^{*} \boldsymbol{o} / C_{[+\mathrm{GRAVE}]}:(6),(29),(30),(51),(94)^{42}$.

$\mathrm{PG}^{*} \boldsymbol{e}, \mathrm{PM}{ }^{*} \boldsymbol{\Lambda} / \boldsymbol{C}_{[+\mathrm{GRAVE}]}:(54),(60)$.

$\mathrm{PG} * \boldsymbol{e} / \boldsymbol{C}_{[+\mathrm{PALATAL}]}, \mathrm{PM}{ }^{*} \boldsymbol{\Lambda}:(77)$.

$\mathrm{PG}^{*} \boldsymbol{e}, \mathrm{PM}^{*} \boldsymbol{a}$ : (25), (80), (87).

PG * $\boldsymbol{e} / \#$, PM Ø: (28), (49), (132).

PG * ${ }^{i}$, PM * $i$ : (6), (9), (15), (21), (32), (42), (52), (56), (64), (68), (72), (85), (96), (100), (103), (112), (115).

PG * $\boldsymbol{i}, \mathrm{PM}{ }^{*} \boldsymbol{e}:(16),(45),(69),(90)$.

$\mathrm{PG}^{*} \boldsymbol{i}, \mathrm{PM}{ }^{*} \boldsymbol{u}$ : (34), (36), (50), (74), (117).

$\mathrm{PG} * \boldsymbol{o} / \boldsymbol{w}_{-}, \mathrm{PM}{ }^{*} \boldsymbol{u}:(93)$.

$\mathrm{PG}^{*} \boldsymbol{o}, \mathrm{PM}$ *o: (8), (11), (58), (62), (66), (88), (89), (94), (128).

PG *o, PM * $\boldsymbol{\Lambda}$ : (1), (15), (18), (19), (23), (28), (31), (32), (38), (46), (53), (54), (57), (67), (73), (79), (87), (123), (133).

$P G{ }^{*} \boldsymbol{o}, \mathrm{PM}^{*} \boldsymbol{e} / \mathrm{j}:(52)$.

PG *o/_\#", PM Ø: (14), (20), (45), (55), (67), (68), (117).

$\mathrm{PG}^{*} \boldsymbol{w} V, \mathrm{PM}{ }^{*} \boldsymbol{u}:(75)$.

41 Cuando ${ }^{*} \boldsymbol{a}$ estaba precedida por ${ }^{*} \boldsymbol{q}$, su desaparición ante pausa se habría producido no solamente en PM sino también en PGS.

42 Hay PG ${ }^{*} \boldsymbol{e}: \mathrm{PM}{ }^{*} \boldsymbol{o}$ en otro contexto en la serie (90), y PG ${ }^{*} \boldsymbol{o} / \boldsymbol{C}_{[+\mathrm{GRAVE}]}: \mathrm{PM}$ * $\boldsymbol{e}$ en la serie (131).

43 Cuando ${ }^{*} \boldsymbol{o}$ estaba precedida originalmente por ${ }^{*} \boldsymbol{k}$, la caída de la vocal final se habría dado no solo en PM sino también, dentro de la familia guaicurú, en PGS. 
Una disimilación por la cual si en un mismo lexema estaban presentes dos consonantes con el rasgo [+GLOTAL], una de ellas lo perdía, habría actuado tanto en guaicurú como -sobre todo- en mataguayo. Esto habría producido disimilaciones entre consonantes glotales y glotalizadas, como las siguientes: pre-PM ${ }^{*}{ }^{*} t^{\prime} . . .2>$ PM ${ }^{\star} t \ldots$. en las series (15) y (74);

pre-PM ${ }^{* *} \boldsymbol{h} . . . t \boldsymbol{s}^{\prime}>\mathrm{PM}{ }^{*} \boldsymbol{h} . . . t \boldsymbol{s}$ en la serie $(16)$;

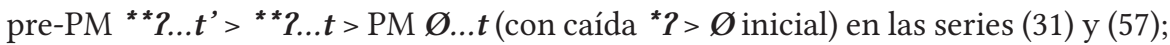

pre-PM ${ }^{*}{ }^{*} t \boldsymbol{s}^{\prime} . . . h>\mathrm{PM}{ }^{*} t \boldsymbol{t} . . . h$ en la serie (37);

pre-PM ${ }^{*} \boldsymbol{t}^{\prime} \ldots \boldsymbol{h}>\mathrm{PM}{ }^{*} \boldsymbol{t} . . . \boldsymbol{h}$ en las series (38) y (46);

pre-PG ${ }^{* *} \boldsymbol{q}^{\prime} \ldots \boldsymbol{w}^{\prime}>\mathrm{PG}{ }^{*} \boldsymbol{q}^{\prime} \ldots \boldsymbol{w}$, pre-PM ${ }^{* *} \boldsymbol{q}^{\prime} \ldots \boldsymbol{h}^{\boldsymbol{w}}>\mathrm{PM}{ }^{*} \boldsymbol{q} \ldots \boldsymbol{h}^{\boldsymbol{w}}$ en la serie (41);

pre-PM ${ }^{* *} t s^{\prime} . . .2>$ PM ${ }^{*} t$ s...? en la serie (43);

pre-PM ${ }^{*} \boldsymbol{h}^{w} \ldots \boldsymbol{t}^{\prime}>\mathrm{PM}{ }^{\star} \boldsymbol{h}^{w} \ldots t$ en las series (50), (90) y (94);

pre-PM ${ }^{*}{ }^{*} \boldsymbol{k}^{\prime} . . . t{ }^{\prime}>\mathrm{PM}{ }^{*} \boldsymbol{k}^{\prime} . . . t$ en la serie $(51)$;

pre-PG ${ }^{* *} q^{\prime} \ldots t^{\prime}>\mathrm{PG}{ }^{*} q \ldots t^{\prime}$, pre-PM ${ }^{* *} q^{\prime} \ldots t^{\prime}>\mathrm{PM} \boldsymbol{q}^{\prime} \ldots t$ en la serie (54);

pre-PG ${ }^{* *} \boldsymbol{m}^{\prime} \ldots \boldsymbol{q}{ }^{\prime}>\mathrm{PG}{ }^{*} \boldsymbol{m}{ }^{\prime} \ldots \boldsymbol{q}$ en la serie (67);

pre-PM ${ }^{*} \boldsymbol{h}^{\boldsymbol{w}} \ldots \boldsymbol{h}>\mathrm{PM}{ }^{*} \boldsymbol{w} . . . \boldsymbol{h}$ en la serie (71);

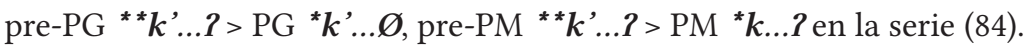

El caso de

pre-PM ${ }^{*}{ }^{*} \boldsymbol{h} . . . t^{\prime} \ldots \boldsymbol{k}^{\prime}>\mathrm{PM}{ }^{\star} \boldsymbol{h} . . . t . . \boldsymbol{k}$ en la serie (91).

supone que el rasgo ${ }_{[+\mathrm{GLOTAL}]}$ se habría mantenido en una de tres consonantes de una misma palabra en las que originalmente habría estado presente.

En las series (51), (56), (70) y (91) hay metátesis en una o más de las lenguas comparadas.

Para la serie (78), es necesario suponer la síncopa de la sílaba inicial en mataguayo.

Es cierto que la reconstrucción de algunos proto-fonemas es discutible. En este trabajo, por ejemplo, he preferido las reconstrucciones PM ${ }^{*} \boldsymbol{k}$ y ${ }^{*} \boldsymbol{q}^{44}$, frente a la sugerencia de Campbell y Grondona (2012) de reconstruir más bien PM ${ }^{*} \boldsymbol{k}^{\boldsymbol{j}}$ (o algún otro tipo de velar anteriorizada) y ${ }^{*} \boldsymbol{k}$, respectivamente. En el estado actual de la comparación, cualquiera de ambas reconstrucciones parece igualmente probable. En cualquier caso, se puede comprobar que las consonantes dorsales de punto de articulación más anterior (velares o, si se prefiere, velares palatalizadas o prevelares) del PM corresponden de manera

44 Las correspondencias entre oclusivas velares del maká y del weenhayek por una parte y entre oclusivas uvulares del maká y del weenhayek, por otra, me parecen decisivas a este respecto. 
sistemática a consonantes velares en PG, mientras que las consonantes dorsales de punto de articulación más posterior (uvulares o, si se prefiere, velares) del PM corresponden también regularmente a consonantes uvulares en PG:

$$
\begin{aligned}
& \mathrm{PG}{ }^{*} \boldsymbol{k}: \mathrm{PM}{ }^{*} \boldsymbol{k}\left(\mathrm{o}^{*} \boldsymbol{k}^{j}\right) \\
& \mathrm{PG}{ }^{*} \boldsymbol{k}^{\prime}: \mathrm{PM}{ }^{*} \boldsymbol{k}^{\prime}\left({ }^{*} \boldsymbol{k}^{j}\right) \\
& \mathrm{PG}{ }^{*} \boldsymbol{q}: \mathrm{PM}{ }^{*} \boldsymbol{q}\left({ }^{*}{ }^{*} \boldsymbol{k}\right) \\
& \mathrm{PG}{ }^{*} \boldsymbol{q}^{\prime}: \mathrm{PM}{ }^{*} \boldsymbol{q}^{\prime}\left({ }^{*}{ }^{*} \boldsymbol{k}^{\prime}\right)
\end{aligned}
$$

Es la regularidad de las correspondencias lo que importa a la hora de comparar los ítems guaicurúes y mataguayos que incluyen reflejos de esos proto-fonemas.

\section{Conclusiones}

Aun cuando la investigación ulterior pueda llegar a invalidar como eventuales cognados a algunas de las semejanzas léxicas presentadas en este trabajo, creo que la cantidad de las mismas es suficiente para contradecir la afirmación, tantas veces repetida ${ }^{45}$, de que existen muy pocas semejanzas en los vocabularios de las lenguas de las familias guaicurú y mataguayo, por oposición a las frecuentes y notables similitudes observables en los sistemas gramaticales. Es cierto que hay pocas semejanzas en algunos campos semánticos específicos (ítems de cultura, zoónimos y fitónimos, términos de parentesco, por ejemplo ${ }^{46}$ ); pero en otros ámbitos (incluyendo verbos, partes del cuerpo, elementos de la naturaleza inanimada) los eventuales cognados parecen ser - por el contrario - abundantes.

Entre lenguas en contacto, la primera parte del vocabulario que pasa en préstamo es - sin excepciones conocidas - el léxico cultural ${ }^{47}$. En el caso guaicurú-mataguayo, la existencia de una cantidad importante de semejanzas en términos del vocabulario básico, no acompañanadas de un número igualmente significativo de similitudes en el vocabulario cultural, coincide con lo que siempre se ha tenido como indicador de probable relación genética ${ }^{48}$.

45 Lafone Quevedo (1896:137-138), Imbelloni (1936:197), Henry (1939:91), Tovar (1951:377378), Campbell y Grondona (2007:3), entre otros muchos.

46 La escasez de semejanzas en estos campos semánticos se puede explicar por diversos factores. En el caso de los zoónimos y fitónimos, por ejemplo, hay que señalar que una cantidad de términos de estos ámbitos son originalmente descriptivos y de creación posiblemente reciente (cf. Messineo y Cuneo 2007, Viegas Barros 2010). Las diferencias en la terminología de parentesco, por su parte, podrían deberse a sucesivas reestructuraciones de los sistemas de parentesco -tanto consanguíneo como político- que habrían ocurrido en la (pre)historia de los distintos pueblos chaqueños.

47 Cf. Thomason y Kaufman (1991: 74): “...we know of no exeptions - and would be astonished to find any - to the rule that nonbasic vocabulary is always borrowed first".

48 Cf. Greenberg (2005 [1953]:15): “The presence of fundamental vocabulary resemblances well beyond chance expectation, not accompanied by resemblances in cultural vocabulary, 
Por otra parte, los eventuales cognados guaicurú-mataguayo están unidos por correspondencias fonológicas bastante regulares. Además, el tipo de semejanzas gramaticales guaicurú-mataguayo no coincide con lo esperado en contextos de difusión sino más bien en situaciones de parentesco, e incluso hay detalles de la alomorfía de algunos morfemas gramaticales que muestran una analogía mayor de lo que a primera vista parecería. Y existen también irregularidades compartidas difíciles de explicar como productos de la difusión.

En resumen, los argumentos a favor de la relación genética guaicurúmataguayo parecen ser fuertes. Por tanto, la hipótesis de parentesco entre ambas famílias parece viable ${ }^{49}$.

Naturalmente, este probable parentesco no sería incompatible con el hecho de que ambas familias puedan formar parte de un área lingüística. Una de las tareas pendientes en la comparación de las lenguas guaicurúes y mataguayas es, precisamente, la profundización de la distinción entre las semejanzas que pueden representar eventuales cognados, y aquellas otras que pueden ser más probablemente - resultado de la difusión lingüística entre las lenguas del Gran Chaco.

\section{Referencias}

Adelaar, Willem F. H. 2012. La importancia de los vocabularios de Johann Natterer (1787-1843) para el conocimiento de las lenguas amazónicas. Ponencia presentada en el Simposio 'Lenguas indígenas de América. Estudios descriptivos-tipológicos y sus contribuciones para la linguística teórica', en el $54^{\circ}$ Congreso Internacional de Americanistas, Viena, 18-19 de julio.

Alvarsson, Jan-Åke. 1984. Wenhayek Lhamet. Introducción al mundo de los matacosnoctenes de Bolivia. Cochabamba: Misión Sueca Libre en Bolivia, 2a. ed. revisada [1a. ed.: 1979].

Arenas, Pastor. 1993. Fitonimia toba-pilagá, Hacia una Nueva Carta Étnica del Gran Chaco, dir. José Braunstein, Las Lomitas (Formosa): Centro del Hombre Antiguo Chaqueño (CHACO), 5:75-100.

Belaieff, Juan. 1940. El macca. Revista de la Sociedad Científica del Paraguay 4(6):1-111. Asunción.

is thus a sure indication of genetic relationship. This is a frequent, indeed normal, situation where a relationship is of a fairly remote order. Pronoun, body parts, etc., will agree while terms like 'pot', 'ax', 'maize', will disagree. The assumption of borrowing here runs contrary to common sense and documented historic facts."

49 En cambio, y en contra de lo sostenido -entre otros- por Greenberg (1987), no parecen existir semejanzas consistentes con una hipótesis de posible parentesco entre las familias guaicurú y/o mataguayo por un lado, y las demás familias lingüísticas chaqueñas (lulevilela, zamuco, enlhet-enenhlet) por otra parte. 
Boggiani, Guido. 1900. Lingüística sudamericana. Datos para el estudio de los idiomas payaguá y machicui. Parte I: Los payaguá. Trabajos de la 4a. Sección del Congreso Científico Latinoamericano, Buenos Aires (separata): 1-38.

Braunstein, José. 1992-1993. Introducción. Hacia una nueva carta étnica del Gran Chaco V. Las Lomitas (Formosa): Centro del Hombre Antiguo Chaqueño (CHACO): 1-3.

Braunstein, José. 1996. Clasificación de las lenguas y pueblos del Gran Chaco. En: Herminia E. Martín y Andrés Pérez Diez (eds.), Lenguas indígenas de Argentina 1492-1992. San Juan: Universidad Nacional de San Juan: 19-32.

Braunstein, José. 2000. Wichí (bazanero) Word-List. Intercontinental Dictionary Series. Vol. I. South American Indian Languages, ed. Mary Ritchie Key, Irvine: University of California. CD-ROM.

Braunstein, José y Herminia E. Martín. 1990-1991. Nuevos rumbos de la etnolingüística chaqueña. Hacia una Nueva Carta Étnica del Gran Chaco II. Las Lomitas (Formosa): Centro del Hombre Antiguo Chaqueño (CHACO): 3-12

Buckwalter, Alberto S. 1980. Vocabulario toba, Buenos Aires: Talleres Grancharoff Hnos.

Buckwalter, Alberto S. \& Lois Litwiller de Buckwalter. 2004. Vocabulario castellanoguaycurú, Formosa/Elkhart: Equipo Menonita/Mennonite Missions Network.

Calvo Pérez, Julio. 1994. Quechua y aimara: lenguas en contacto. Estudios de Lengua y Cultura Amerindias I (Actas de las II Jornadas Internacionales de Lengua y Cultura Amerindias), Noviembre, 1993. Valencia: 95-112.

Campbell, Lyle. 2006. Contacto lingüístico entre las lenguas del Gran Chaco. ¿Existe un Área Lingüística Chaqueña? Ponencia presentada en el Simposio 'Avances en Lingüística Histórico-Comparativa Aborigen Sudamericana' en el $52^{\circ}$ Congreso Internacional de Americanistas, Sevilla: Universidad de Sevilla. 17-18 de julio de 2006.

Campbell, Lyle y Verónica Grondona. 2007. Internal Reconstruction in Chulupí (Nivaclé). Diachronica 24:1-29.

Campbell, Lyle y Verónica Grondona. 2012. Languages of the Gran Chaco and the Southern Cone. Lyle Campbell y Verónica Grondona (eds.), The indigenous languages of South America: A comprehensive guide, p. 625-668. Berlin/Boston: De Gruyter Mouton

Carol, Javier. 2011. Determinantes demostrativos en chorote (mataguayo). Interrelación con la modalidad, la temporalidad y la evidencialidad, Indiana, 28:227-254. Berlín: Ibero-Amerikanisches Institut Preussicher Kulturbesitz.

Carpio, M. Belén. 2012. Fonología y morfosintaxis de la lengua hablada por grupos tobas en el oeste de Formosa (Argentina). Münich: LINCOM. LINCOM Studies in Native American Languages (LSNAL) $n^{\circ} 67$.

Castelnau, Francis de. 1851. Expédition dans les parties centrales de l'Amérique du Sud: de Rio de Janeiro à Lima, et de Lima au Para, executée par ordre du Gouvernement français pendant les années 1843 à 1847. Histoire du Voyage, París: P. Bertrand, v. 5. 
Censabella, Marisa. 2002. Descripción funcional de un corpus en lengua toba. Fonología, clases sintácticas y derivación. Aspectos de sincronía dinámica. Tesis de Doctorado en Letras Modernas. Córdoba: Universidad Nacional de Córdoba.

Censabella, Marisa. 2009. Sistema fonológico y sincronía dinámica de seis variedades orientales del continuum wichí. En: Ana Fernández Garay y Marisa Censabella (eds.), Estudios fonológicos de continua dialectales: mapuche y wichí. Santa Rosa: Universidad Nacional de La Pampa, Facultad de Ciencias Humanas: 99-133.

Censabella, Marisa y Jimena Terraza. 2010. Aplicativos en toba y wichí: forma y función. En: Marisa Censabella \& Raúl González (eds.), Libro de Actas II de Lenguas Indígenas Americanas y II Simposio Internacional de Lingüística Amerindia, ALFAL, 17-19 de septiembre de 2009, Resistencia, Chaco. Buenos Aires: CONICET (CD-ROM).

Ceria, Verónica G. y Filomena Sandalo. 1995. A preliminary reconstruction of Proto-Waikurúan with special reference to pronominals and demonstratives, Anthropological Linguistics, Bloomington, 37 (2):169-191.

Claesson, Kenneth. 1994. A phonological outline of mataco noctenes. International Journal of American Linguistics 60 (2):1-38.

Comrie, Bernard, Lucía A. Golluscio, Hebe González y Alejandra Vidal. 2010. El Chaco como área lingüística. En Zarina Estrada Fernández y Ramón Arzápalo Marín (Eds.) Estudios de lenguas amerindias 2: Contribuciones al estudio de las lenguas originarias de América. Hermosillo: Unison.

Cúneo, Paola, Patricia Dante y Temis Tacconi. 2009. Tipología léxica. Una aproximación a dos lenguas chaqueñas: toba (guaycurú) y maká (mataguaya), Cadernos de Etnolingüística 1(2) < http://etnolinguistica.wdfiles.com/local--files/ vol1\%3A2/cadernos_vol1_n2.pdf>Consultado el 15/08/2011.

Drayson, Nicolás. 2009. Diccionario Iyojwa’ja 'Lij - Kilay 'Lij (Chorote-Castellano). Hacia una Nueva Carta Étnica del Gran Chaco VIII, dir. José Braunstein, Las Lomitas (Formosa): Centro del Hombre Antiguo Chaqueño (CHACO):91-174.

Fabre, Alain. 2005. Los pueblos del Gran Chaco y sus lenguas. Segunda parte: Los mataguayo. Suplemento Antropológico 40(14):313-435 Asunción: Universidad Católica 'Nuestra Señora de la Asumción' / Centro de Estudios Antropológicos. Disponible online en Diccionario etnolingüístico y guía bibliográfica de los pueblos indígenas sudamericanas, Versión electrónica. <http://butler.cc.tut.fi/ fabre/ BookInternetVersio/Dic=Mataguayo.pdf>. Cpmsultado el 15/08/2011.

Fabre, Alain. 2006. Los clasificadores posesivos en las lenguas mataguayo, guaykurú, enlhet-enenlhet ("lengua-maskoy"), zamuco, vilela y besïro (chiquitano). Semejanzas y diferencias desde una perspectiva areal chaqueña. Ponencia al $52^{\circ}$ Congreso Internacional de Americanistas, Sevilla, 17 al 21 de julio.

Fabre, Alain. 2007. Morfosintaxis de los clasificadores posesivos en las lenguas del Gran Chaco (Argentina, Bolivia y Paraguay. UniverSOS. Revista de Lenguas Indígenas y Universos Culturales 4:67-85.

Gerzenstein, Ana. 1979. Lengua chorote. Buenos Aires: Universidad de Buenos Aires, Facultad de Filosofía y Letras, Instituto de Lingüística (Archivos de Lenguas Precolombinas, 3). Vol. 2. 
Gerzenstein, Ana. 1983. Lengua chorote. Variedad 2. Buenos Aires: Universidad de Buenos Aires, Facultad de Filosofía y Letras, Instituto de Lingüística (Archivos de Lenguas Precolombinas, 4).

Gerzenstein, Ana. 1987. The Chorote language: three examples of internal reconstruction. Language Science 9(1):11-15.

Gerzenstein, Ana. 1995. El maká. Estudio descriptivo. Buenos Aires: Universidad de Buenos Aires, Facultad de Filosofía y Letras, Instituto de Lingüística (Colección 'Nuestra América'. Serie: Archivo de Lenguas Indoamericanas).

Gerzenstein, Ana. 1999. Diccionario etnolingüístico maká-español. Índice españolmaká. Buenos Aires: Universidad de Buenos Aires, Facultad de Filosofía y Letras, Instituto de Lingüística (Colección 'Nuestra América'. Serie: Archivo de Lenguas Indoamericanas).

Gerzenstein, Ana. 2003 Variaciones dialectales de algunas unidades del sistema consonántico wichí. En: A. Tissera de Molina y J. Zigarán (eds.), Lenguas y culturas en contacto: 69-81. Salta: Universidad Nacional de Salta.

Gerzenstein, Ana y Beatriz Gualdieri. 2003. La armonía vocálica en lenguas chaqueñas de las familias guaycurú y mataguaya. LIAMES 3:99-118. Campinas, Unicamp.

Greenberg, Joseph H. 1987. Language in the Americas, Stanford: Stanford University Press.

Greenberg, Joseph H. 2005. Historical linguistics and unwritten languages. Genetic Linguistics. Essays in Theory and Method: 3-31. Oxford: Oxford University Press (ed. original 1953).

Griffiths, Glynn. 2002. Dicionário da língua kadiwéu. Kadiwéu - Portugués, Português - Kadiwéu. Cuiabá: Sociedade Internacional de Lingüística.

Grondona, Verónica M. 1998. A Grammar of Mocoví. Puttsburgh: University of Pittsburgh: unpublished $\mathrm{Ph}$. D dissertation.

Grondona, Verónica M. 2006. El estado actual de reconstrucción comparativa en dos familias de lenguas chaqueñas. Ponencia presentada en el Simposio 'Avances en Lingüística Histórico-Comparativa Aborigen Sudamericana' en el $52^{\circ}$ Congreso Internacional de Americanistas, Sevilla: Universidad de Sevilla. 17-18 de julio de 2006.

Gualdieri, Beatriz. 1998. Mocovi (Guaicuru). Fonologia e morfosintaxe. Tese de Doutorado. Campinas, SP: UNICAMP.

Henry, Jules. 1939. The linguistic position of the Ashluslay Indians, International Journal of American Linguistics 10 (2-3): 86-91.

Hunt, Richard J. 1913. El vejoz o aiyo. Introducción de S. A. Lafone Quevedo, Revista del Museo de La Plata 22:7-214. (2 ${ }^{\text {a }}$ Serie, 9), Buenos Aires.

Hunt, Richard J. 1915. El chorotí o yófuaja, con vocabularies enimaga o towothli y chunupi o suhin, Revista del Museo de La Plata 23 (2a . Serie, 10), Liverpool.

Imbelloni, José. 1936. Lenguas indígenas del territorio argentino. Historia de la Nación Argentina 1:177-205. Buenos Aires: Junta de Historia y Numismática Americana. 
Kaufman, Terrence. 1990. The native languages of South America. What we know and how to know more, Amazonian linguistics. Studies in lowland South American Languages, ed. by Doris L. Payne, Austin: University of Texas Press:13-74.

Kaufman, Terrence. 1994. Language history in South America, Atlas of the world's languages. Ed. Christopher Moseley y R. E. Asher, Londres: Routledge:46-76.

Kirtchuk, Pablo I. 1996. Lingüística areal: deixis y clasificación nominal en lenguas del Gran Chaco. En: Herminia Martín y Andrés Pérez Diez (comps.), Lenguas indígenas de Argentina. San Juan: Ed. Fundación Universidad Nacional de San Juan:75-85.

Koch-Grünberg, Theodor. 1903. Die Guaikurú Gruppe, Mitteilungen der anthropologischen Gesellschaft in Wien 33:1-134. Viena.

Lafone Quevedo, Samuel A. 1892-1893. La lengua mocoví según el padre Francisco Tavolini. MS en la Biblioteca del General Bartolomé Mitre. Con introducción y notas por... Revista del Museo de La Plata 1:71-112, 256-304 y 2: 175-224, 425-460.

Lafone Quevedo, Samuel A. 1894-1896. Arte de la lengua toba por el padre Alonso Bárcena Soc. Jes. (MS propiedad del General B. Mitre) con vocabularios facilitados por los Sres. Dr. Angel Carranza, Pelleschi, y otros. Editados y comentados por... Revista del Museo de La Plata 5:93-184 y 7:191-227.

Lafone Quevedo, Samuel A. 1896a. Grupo mataco-mataguayo del Chaco: dialecto Vejoz. Ms. d'Orbigny. Boletín del Instituto Geográfico Argentino 17:127-176. Buenos Aires.

Lafone Quevedo, Samuel A. 1896b. Vocabulario castellano-toba por el padre Alonso Bárcena (Ms en la biblioteca del General Mitre) acompañado de equivalencias apuntadas de boca del indio López en 1888. Revista del Museo de La Plata 7:229-261.

Mason, J. Alden. 1950. The Languages of South American Indians.Handbook of South American Indians, ed. por Julian H. Steward, 6 (Bulletin 149, Bureau of American Ethnology), Washington:157-317.

Messineo, Cristina. 2002. El contraste alienable/inalienable en las construcciones posesivas del toba (guaycurú), Temas de Lingüística Aborigen II, coords. Ana Fernández Garay y Lucía Golluscio, Buenos Aires: Universidad de Buenos Aires, Facultad de Filosofía y Letras, Instituto de Lingüística (Serie Archivo de Lenguas Indoamericanas, Colección Nuestra América):53-81.

Messineo, Cristina. 2003. Toba (guaycurú). Aspectos gramaticales y discursivos. (Lincom Studies in Native American Languages). Muenchen: LINCOM EUROPA.

Messineo, Cristina. 2008. La negación en toba (guaycurú) y en maká (matacomataguayo), Aproximación tipológica a las lenguas del Chaco. Ponencia presentada en el II Simposio Internacional de Documentación Lingüística y Cultural en América Latina “Contacto de Lenguas y Documentación”, Buenos Aires: Biblioteca Nacional, 14-15 de agosto de 2008.

Messineo, Cristina. 2011. Aproximación tipólogica a las lenguas indígenas del Gran Chaco. Rasgos compartidos entre toba (familia guaycurú) y maká (familia matacomataguayo), Indiana 28:183-225. Berlín: Ibero-Amerikanisches Institut Preussicher Kulturbesitz. 
Messineo, Cristina y Paola Cúneo. 2007. Recursos lingüísticos para la clasificación de plantas y animales en dos lenguas indígenas del Chaco (toba y maká). Proceedings of the III Conference on Indigenous Languages of Latin America. 25-27 de octubre de 2007. Austin: University of Texas, Center for Indigenous Languages of Latin America (CILLA) / Teresa Lozano Long Institute of Latin American Studies. <http:// www.ailla.utexas.org/site/cilla3/Messineo-Cuneo_CILLA_III.pdf>. Consultado el 15/08/2011.

Messineo, Cristina y Paola Cúneo. 2010. Modos de clasificación nominal en toba (guaycurú) y maká (mataguaya). Léxico, clasificación nominal y categorización etnobiológica en el Gran Chaco. Cristina Messineo, Gustavo Scarpa \& Florencia Tola (comp.). Santa Rosa: Universidad Nacional de La Pampa, Facultad de Ciencias Humanas, Instituto de Lingüística: 27-64.

Messineo, Cristina y Ana Gerzenstein. 2007. La posesión en dos lenguas indígenas del Gran Chaco: toba (guaycurú) y maká (mataguayo). LIAMES (Revista de lingüística indígena americana) 7:61-79. Campinas: Universidade Estadual de Campinas, IEL.

Messineo, Cristina, Javier Carol y Harriet Manelis Klein. 2012. Los determinantes demostrativos en las lenguas guaycurúes y mataguayas (Región del Gran Chaco). Memorias del $V$ Congreso de Idiomas Indígenas de Latinoaméria, 6-8 de octubre de 2011. Austin: University of Texas, Center for Indigenous Languages of Latin America (CILLA) / Teresa Lozano Long Institute of Latin American Studies. $<$ http://www.ailla.utexas.org/site/events/html>. Consultado el 23/02/2012.

Mitre, Bartolomé.1910. Vocabulaure Yuiagdech, ou des Lenguas, tribu presqu' inconnue du Grand Chaco. Etabliée sur les bord du rio Pilcomayo, près de la ville de l'Assomption du Paraguai, avec un avant-propos par don Pedro de Angelis [Pedro Cerviño, 1839]. Catálogo razonado de la sección lenguas americanas del Museo Mitre 2:142-169. Buenos Aires, Coni Hermanos.

Najlis, Elena L. 1966. Lengua abipona, Buenos Aires: Universidad de Buenos Aires, Facultad de Filosofía y Letras, Centro de Estudios Lingüísticos, Archivo de Lenguas Precolombinas, $\mathrm{N}^{\circ}$. 1, 2 vol.

Najlis, Elena L. 1971. Pre-mataco phonology. International fournal of American Linguistics 37(2):128-130.

Najlis, Elena L. 1984. Fonología de la protolengua mataguaya. Buenos Aires: Universidad de Buenos Aires, Facultad de Filosofía y Letras, Instituto de Lingüística (Cuadernos de Lingüística Indígena, 9).

Nonato, Rafael y Filomena Sandalo. 2007. Uma comparação gramatical, fonológica e lexical entre as famílias Guaikurú, Mataco e Bororo: um caso de difusão areal? Boletim do Museo Paraense Emílio Goeldi, Ciências Humanas 2(14):91-113. Belém.

Paucke, Florian. 1942-1944. Hacia acá y para allá (Una estadía entre los indios mocobies, 1749-1767). Traducción castellana por Edmundo Wernicke. Advertencia por Radamés A. Altieri. Tucumán-Buenos Aires: Universidad Nacional de Tucumán, Departamento de Investigaciones Regionales, Instituto de Antropología / Institución Cultural Argentino-Germana. 4 volúmenes. 
Salanova, Andrés y Javier Carol. 2011. On pseudo-applicatives. <http://aix1.uottawa. ca/ asalanov/Docs/pseudo-applicatives.pdf>. Consultado el 23/02/2012.

Sánchez Labrador, José. 1971. Vocabulario Eyiguayegi. Según el manuscrito del siglo XVIII. Parte 1ra: Letras: A - LL. (Lenguas Chaqueñas. Tomo III, vol. 4. Edición: B. Sušnik, Asunción: Museo Etnográfico 'Andrés Barbero’.

Sánchez Labrador, José. 1972. Vocabulario Eyiguayegi. Según el manuscrito del siglo XVIII. Parte 2da: Letras: $M-Z$. (Lenguas Chaqueñas. Tomo III, vol. 5. Edición: B. Sušnik, Asunción: Museo Etnográfico ‘Andrés Barbero'.

Sandalo, Filomena. 1997. A grammar of Kadiweu with special reference to the Polysynthesys Parameter, Cambridge: MIT (MIT Occasional Papers in Linguistics, 11).

Schmidt, Max. 1940. Los payaguá, Revista do Museu Paulista, 3, So Pablo: 135-317.

Seelwische, José. 1975. Na Lhasinônash napi Nivacle. Gramática Nivacle. Asunción: "El Gráfico” S. R. L.

Stell, Nélida N. 1989. Gramática descriptiva de la lengua Niwaklé (Chulupí). Tesis para optar al título de Doctor. Buenos Aires: Universidad de Buenos Aires, Facultad de Filosofía y Letras. 2 vols.

Stell, Nélida N. y José Seelwische. 2000. Nivaclé Word-List. Intercontinental Dictionary Series. Vol. I. South American Indian Languages, ed. Mary Ritchie Key, Irvine: University of California. CD-ROM.

Suárez, Jorge A. 1988. Lenguas indígenas de América del Sur, Estudios sobre lenguas indígenas sudamericanas, Bahía Blanca: Universidad Nacional del Sur: 13-30, traducción de Silvia Suardiaz de Antollini y Elizabeth M. Rigatuso (originalmente: South American Indian Languages, en Encyclopaedia Britannica, 15a. ed.: 111-12 [1974]).

Swadesh, Mauricio. 1959. Mapas de Clasificación Lingüística de México y las Américas, México: Universidad Nacional Autónoma de México, Cuadernos del Instituto de Historia, Serie Antropológica, 8.

Tovar, Antonio. 1951. Un capítulo de lingüística general. Los prefijos personales en lenguas del Chaco y la lucha entre préstamos morfológicos en un espacio dado. Boletín de la Academia Argentina de Letras, año 20, 77:369-403.

Tovar, Antonio. 1961. Catálogo de las lenguas de América del Sur. Buenos Aires: Sudamericana.

Tovar, Antonio. 1964. Relación entre las lenguas del grupo mataco, Homenaje a Fernando Márquez-Miranda, arqueólogo e historiador de América. Sevilla: 370-377.

Tovar, Antonio. 1981. Relatos y diálogos de los matacos, seguidos de una gramática de su lengua. Madrid: Ediciones Cultura Hispánica del Instituto de Cooperación Iberoamericana.

Tovar, Antonio y Consuelo Larrucea de Tovar. 1984. Catálogo de las lenguas de América del Sur. Madrid: Gredos. 
Vidal, Alejandra. 2001. Pilagá Grammar (Guaykuruan Family, Argentina), University of Oregon. PhD. dissertation.

Vidal, Alejandra. 2006. Aplicativos en pilagá (guaycurú) y wichí (mataco-mataguayo): un estudio comparativo. Ponencia presentada en el Simposio 'Lenguas chaqueñas en Argentina, Bolivia y Paraguay: estudios descriptivos, tipológicos y comparativos' en el $52^{\circ}$ Congreso Internacional de Americanistas, Sevilla: Universidad de Sevilla. 20-21 de julio de 2006.

Vidal, Alejandra. 2011. Descripción y explicación en el estudio de dos lenguas chaqueñas: pilagá (guaycurú) y wichí (mataguaya). RASAL Lingüística (Revista de la Sociedad Argentina de Lingüística) 2010, v: 1-2: 141-155. Buenos Aires: Instituto de Filología y Literaturas Hispánicas "Dr. Amado Alonso", Fac. de Filosofía y Letras, UBA.

Vidal, Alejandra y Analía Gutiérrez. 2010. La categoría de 'tiempo nominal' en las lenguas chaqueñas, En Víctor M. Castel y Liliana Cubo de Severino (Ed), La renovación de la palabra en el bicentenario de la Argentina. Los colores de la mirada lingüística. Mendoza: Editorial FFyL, UNCuyo.: <http://mendoza-conicet.gob.ar/ institutos/incihusa/ul/csal12/Vidal_195_CSAL12.pdf>. Consultado el 23/02/2012.

Viegas Barros, J. Pedro. 1992-1993. ¿Existe una relación genética entre las lenguas mataguayas y guaycurúes?, en J. Braunstein (ed.), Hacia una nueva carta étnica del Gran Chaco V, Las Lomitas (Formosa): Centro del Hombre Antiguo Chaqueño (CHACO): 193-213.

Viegas Barros, J. Pedro. 2002. Fonología del proto-mataguayo: las fricativas dorsales. Current Studies on South American Languages, ILLA (Indigenous Languages of Latin America), Vol. 3. Contribuciones seleccionadas del 50 Congreso Internacional de Americanistas en Varsovia y del Taller Spinoza de Lenguas Amerindias en Leiden (2000), Leiden: Research School of Asian, African, and Amerindian Studies (CNWS): 137-148.

Viegas Barros, J. Pedro. 2004. Guaicurú no, macro-guaicurú sí. Una hipótesis sobre la clasificación de la lengua guachí (Mato Grosso do Sul, Brasil), Biblioteca Virtual, Grupo Etnolingüística, Goiánia: Universidade de Goiás, <http://br.groups.yahoo.com/group/etnolinguistica/files/Biblioteca_Virtual/ GuachiMacroGuaikuruViegasBarros.PDF> Consultado el 15/08/2011.

Viegas Barros, J. Pedro. 2006. La hipótesis macro-guaicurú. Semejanzas gramaticales guaicurú-mataguayo. Revista UniverSOS, 3: 183-212. Valencia: Universidad de Valencia.

Viegas Barros, J. Pedro. 2008. Consonantes glotalizadas en proto-guaicurú. Estudios en Lingüística y Antropología. Homenaje a Ana Gerzenstein. Cristina Messineo, Marisa Malvestitti y Roberto Bein (eds.). Buenos Aires: Universidad de Buenos Aires, Facultad de Filosofía y Letras: 59-66.

Viegas Barros, J. Pedro. 2010. La etimología de ‘tapir' en guaicurú. Léxico, clasificación nominal y categorización etnobiológica en el Gran Chaco. Cristina Messineo, Gustavo Scarpa \& Florencia Tola (comp.). Santa Rosa: Universidad Nacional de La Pampa, Facultad de Ciencias Humanas, Instituto de Lingüística: 141-156. 
Viegas Barros, J. Pedro. 2012. De verbo a marca de persona. El origen del prefijo de segunda persona ${ }^{*} \boldsymbol{m} \boldsymbol{V}$ - en la lengua maká. Ponencia presentada en el Simposio Hechos y datos de los pueblos del Gran Chaco: diálogo histórico entre la antropología, la sociología, la lingüística, y la arqueología para un esclarecimiento del estado actual de las poblaciones originarias de la región, en el $54^{\circ}$ Congreso Internacional de Americanistas, 17-18 de julio. Viena: Universidad de Viena.

Viegas Barros, J. Pedro. 2013. Proto-guaicurú. Una reconstrucción fonológica, léxica y morfológica. Münich: LINCOM. LINCOM Studies in Native American Languages (LSNAL) 69.

Viegas Barros, J. Pedro. En prensa. Las consonantes africadas del proto-guaicurú. En: Hebe González y Beatriz Gualdieri (eds.). Actas XIII Congreso de la Sociedad Argentina de Lingüística. Homenaje a Berta Vidal de Battini, Coloquio Lenguas Indígenas I: Sistemas fonológicos de lenguas indígenas de América del Sur: descripción, tipología y contacto. Buenos Aires: Sociedad Argentina de Lingüística.

Viñas Urquiza, María T. 1974. Lengua mataca. Buenos Aires: Universidad de Buenos Aires, Facultad de Filosofía y Letras, Centro de Estudios Lingüísticos (Archivos de Lenguas Precolombinas, 2). 2 vol.

Data recebimento: 01/10/2012

Data aceite: 15/08/2013 